\title{
FILTER-INDUCED BIAS IN Ly $\alpha$ EMITTER SURVEYS: A COMPARISON BETWEEN STANDARD AND TUNABLE FILTERS. GRAN TELESCOPIO CANARIAS PRELIMINARY RESULTS
}

\author{
J. A. De Diego ${ }^{1,7}$, M. A. De Leo ${ }^{1}$, J. CePa ${ }^{2,3}$, A. Bongiovanni ${ }^{2,3}$, T. Verdugo ${ }^{4}$, \\ M. SÁNCHEZ-PORTAL ${ }^{5,8}$, AND J. I. GonZÁlez-SERRANO ${ }^{6}$ \\ ${ }^{1}$ Instituto de Astronomía, Universidad Nacional Autónoma de México Avenida Universidad 3000, Ciudad Universitaria, \\ C.P. 04510, Distrito Federal, Mexico; jdo@astro.unam.mx \\ 2 Instituto de Astrofísica de Canarias, E-38205 La Laguna, Tenerife, Spain \\ ${ }^{3}$ Departamento de Astrofísica, Universidad de La Laguna, E-38206 La Laguna, Tenerife, Spain \\ ${ }^{4}$ Centro de Investigaciones de Astronomía (CIDA), Apartado Postal 264, Mérida 5101-A, Venezuela \\ ${ }^{5}$ Herschel Science Centre (HSC), European Space Agency Centre (ESAC)/INSA, Villanueva de la Cañada, Madrid, Spain \\ ${ }^{6}$ Instituto de Física de Cantabria (CSIC-Universidad de Cantabria), E-39005 Santander, Spain \\ Received 2012 June 15; accepted 2013 August 2; published 2013 September 12
}

\begin{abstract}
Ly $\alpha$ emitter (LAE) surveys have successfully used the excess in a narrowband filter compared to a nearby broadband image to find candidates. However, the odd spectral energy distribution (SED) of LAEs combined with the instrumental profile has important effects on the properties of the candidate samples extracted from these surveys. We investigate the effect of the bandpass width and the transmission profile of the narrowband filters used for extracting LAE candidates at redshifts $z \simeq 6.5$ through Monte Carlo simulations, and we present pilot observations to test the performance of tunable filters to find LAEs and other emission-line candidates. We compare the samples obtained using a narrow ideal rectangular filter, the Subaru NB921 narrowband filter, and sweeping across a wavelength range using the ultra-narrow-band tunable filters of the instrument OSIRIS, installed at the $10.4 \mathrm{~m}$ Gran Telescopio Canarias. We use this instrument for extracting LAE candidates from a small set of real observations. Broadband data from the Subaru, Hubble Space Telescope, and Spitzer databases were used for fitting SEDs to calculate photometric redshifts and to identify interlopers. Narrowband surveys are very efficient in finding LAEs in large sky areas, but the samples obtained are not evenly distributed in redshift along the filter bandpass, and the number of LAEs with equivalent widths $<60 \AA$ can be underestimated. These biased results do not appear in samples obtained using ultra-narrow-band tunable filters. However, the field size of tunable filters is restricted because of the variation of the effective wavelength across the image. Thus, narrowband and ultra-narrow-band surveys are complementary strategies to investigate high-redshift LAEs.
\end{abstract}

Key words: galaxies: high-redshift - methods: data analysis - techniques: miscellaneous

Online-only material: color figure

\section{INTRODUCTION}

The odd spectral energy distribution (SED) of Ly $\alpha$ emitter galaxies (LAEs) convolved with the instrumental profile can introduce unsought biases on the characteristics of the candidate samples extracted from photometric surveys. Particularly, properties such as the equivalent width (EW) and the redshift distribution of the subsequently spectroscopically confirmed LAEs may be easily affected by the photometric survey instrumentation and methodology used. On the other hand, these surveys yield reliable photometric redshifts for LAEs and Lyman break galaxies (LBGs), and thus they are useful cosmological tools, as these objects trace dark matter halos and subsequently the evolution of matter distribution in the universe. Furthermore, LAEs at $z \geqslant 6.5$ are also important to study the last stages of the reionization epoch (Malhotra \& Rhoads 2004; Kashikawa et al. 2006; Shibuya et al. 2012). Therefore, it is important to develop unbiased alternative strategies to find new LAE candidate samples.

LBG candidates are selected using the drop-out technique, which consists in comparing images of the galaxy obtained in several broadband filters that cover contiguous wavelength ranges to both sides of the Lyman break at $912 \AA$ (Steidel et al.

\footnotetext{
7 Visiting Astronomer, Instituto de Astrofísica de Canarias.

8 Asociación ASPID, Apartado de Correos 412, La Laguna, Tenerife, Spain.
}

1996). In a similar way, LAE candidates are usually selected by an excess in a narrowband filter compared to a nearby broadband image (Cowie \& Hu 1998). The latter technique is routinely used to find LAE candidates in the Subaru Deep Field (Taniguchi et al. 2005; Kashikawa et al. 2006, 2011) and the Subaru/ XMM-Newton Deep Survey Field (Ota et al. 2010; Ouchi et al. 2010; Shibuya et al. 2012). Narrowband filters have bandwidths of FWHM $\sim 100 \AA$, and to avoid atmospheric $\mathrm{OH}$ emission lines, narrowband surveys are confined to a limited number of redshift ranges. An important result of the narrowband surveys is that the luminosity function (LF) of LAEs remains constant between redshifts $3.0 \leqslant z \leqslant 5.7$, but evolves dramatically between $5.7 \leqslant z \leqslant 6.5$, and maybe beyond (Pentericci et al. 2011; Hibon et al. 2012; Ota \& Iye 2012).

Despite the utility of narrowband filters to find LAE candidates, some groups have used ultra-narrow-band filters to perform this task. Thus, Tilvi et al. (2010) and Krug et al. (2012) have employed a filter with an FWHM $\sim 9 \AA$, installed on the $4 \mathrm{~m}$ Mayall Telescope, seeking LAEs at redshift $z=7.7$; they expected to find one or two LAEs, respectively, but instead found four LAE candidates, and argued about a possible lack of evolution of the LAE LF for redshifts from 3.1 to 8. Recently, Swinbank et al. (2012) searched for LAEs around two quasars at $z \sim 2.2$ and one quasar at $z \sim 4.5$ with the Taurus Tunable Filter instrument installed on the $3.9 \mathrm{~m}$ Anglo-Australian Telescope, using a bandpass of FWHM $=10 \AA$, and found a local 
number density an order of magnitude higher than what might be expected in the field.

The spectra of LAEs are characterized by an asymmetric Ly $\alpha$ line profile with a steep blue cutoff due to absorption by neutral hydrogen, as shown in Figure 3 in $\mathrm{Hu} \&$ Cowie (2006). At redshifts $z>6$, hydrogen completely suppresses the continuum of the galaxy at the blue side of the line (Gunn-Peterson trough), while there is a dim continuum at the red side. Narrowband-selected LAEs usually have high line luminosities $\left(L_{\alpha}>1.5 \times 10^{42} \mathrm{erg} \mathrm{s}^{-1}\right)$ and large line EWs at rest $\left(\mathrm{EW}_{0} \geqslant 20 \AA\right.$ A, e.g., Dayal \& Ferrara 2012; Mallery et al. 2012), although the actual limits may substantially vary because of technical constraints and selection strategies (cf. Hayes et al. 2010; Ouchi et al. 2008, 2010). These properties provide clues which suggest that LAEs represent an early stage of a starburst in an interstellar medium of very low metallicity and almost free of dust, with a radius of about $2 \mathrm{kpc}$ and star formation rates around $6 M_{\odot} \mathrm{yr}^{-1}$ (Taniguchi et al. 2005).

The fraction of Ly $\alpha$ photons that escape from a high-redshift star-forming region is still an active and open topic, as well as an important parameter to account for the observable properties of LAEs. Neutral hydrogen resonantly scatters the Ly $\alpha$ photons, changes their escape paths, and hinders the realization of the photons that are absorbed by dust. Ono et al. (2010) place an upper limit of $20 \%$ escaping photons at $z=6.6$, but at lower redshifts Blanc et al. (2011) and Ciardullo et al. (2012) find that the photon escape fraction may be as high as $100 \%$. Hayes et al. (2010) consider the Ly $\alpha$ luminosity density at $z=2$ significantly underestimated from its intrinsic value, due to the fact that only 1 in 20 photons, on average, reach the telescope; this will especially happen at $z>6$, where the neutral fraction of the intergalactic medium may cause significant suppression of the Ly $\alpha$ line (Santos et al. 2004; Hayes \& Östlin 2006; Dijkstra et al. 2007). On the other hand, $\operatorname{Ly} \alpha$ photons may be less attenuated than non-resonant radiation under suitable conditions within a multiphase scattering medium (Neufeld 1991; Hansen \& Oh 2006; Finkelstein et al. 2008). For Finkelstein et al. (2011), dust geometry shapes the SEDs and has a major influence in the observed EW at the rest frame of the $\operatorname{Ly} \alpha$ line $(\mathrm{EW}(\operatorname{Ly} \alpha))$ distribution in high-redshift LAEs. Finally, the presence of outflows can increase the fraction of Ly $\alpha$ photons that escape from the star-forming region (Kunth et al. 1998; Tapken et al. 2007; Verhamme et al. 2006, 2008; Atek et al. 2008, 2009).

If the observational aspects of LAEs depend on geometrical effects (dust spatial distribution), orientation, and the presence of outflows, the separation between LBGs and LAEs becomes somewhat diffuse. Thus, Kashikawa et al. (2007) classify galaxies with contrasts as low as $\operatorname{EW}(\operatorname{Ly} \alpha)>10 \AA$ as LAEs, while recently Krug et al. (2012) consider even lower limits $(\mathrm{EW}(\mathrm{Ly} \alpha) \gtrsim 4.8 \AA)$ for $z \sim 7.7$ LAEs. Besides, other observations indicate that the fraction of low ultraviolet luminosity LBGs $\left(M_{\mathrm{UV}}>-20.5\right)$ with $\mathrm{EW}(\mathrm{Ly} \alpha)>50 \AA$ is about one-half (Stark et al. 2010, 2011; Pentericci et al. 2011; Schenker et al. 2012; Vanzella et al. 2011; Ono et al. 2012). The presence of $\mathrm{Ly} \alpha$ lines in some LBGs provides strong evidence of the connection between these objects and LAEs. Eventually, this connection will promote an effort to build theoretical models to explain the underlying physics of LAEs and LBGs (e.g., Shapley et al. 2001; Dayal \& Ferrara 2012; Forero-Romero et al. 2012).

We are conducting a blind search on selected gravitationally lensing galaxy cluster sky fields to obtain samples of LAEs not subjected to possible biases imposed by the methodology of narrowband filters. Hence, we are obtaining ultra-narrow-band images using tunable filters of the instrument OSIRIS (OSIRISTF) attached to the $10.4 \mathrm{~m}$ Gran Telescopio Canarias (GTC). OSIRIS-TF is optimized for line flux determination and thus can be called a Star Formation Machine (Cepa et al. 2003). This characteristic makes OSIRIS-TF a powerful instrument to detect faint young galaxies with accurate photometric redshift estimates. Therefore, apart from LAEs, we also expect to obtain other high-redshift candidates, such as LBGs, active galactic nuclei, and line emitters.

This paper is organized as follows. Section 2 introduces the bias produced by the filter bandwidth on the photometric selection of LAEs. Section 3 describes the generation of Monte Carlo data for simulated LAE spectra. Section 4 presents the LAE recovered samples obtained using narrowband filters on the simulated data, exemplified by an ideal rectangular filter and the NB921 Subaru filter. Section 5 describes the methodology used to search for LAE candidates using OSIRIS-TF and presents the sample recovered from the simulated data. Our methodology is then applied on a pilot observation of real data obtained at the GTC to select preliminary candidates at redshifts $z \simeq 6.5$. In Section 6 we cross-check our OSIRIS-TF preliminary candidates with photometric archive data and models of galaxies at high redshift to study the SED and to improve classification. Section 7 presents the discussion of the results obtained with simulated and real data. Finally, Section 8 presents our conclusions.

Throughout this paper we assume a cosmological model with $H_{0}=70 \mathrm{~km} \mathrm{~s}^{-1} \mathrm{Mpc}^{-1}(h=0.7), \Omega_{\mathrm{M}}=0.27$, and $\Omega_{\Lambda}=0.73$.

\section{THE ASYMMETRIC CONTINUUM BIAS}

The spectrum of an LAE at $z \sim 6.5$ shows a sharp decay due to the absorption of $\operatorname{Ly} \alpha$ photons by neutral hydrogen that truncates the blue part of the emission line. From the observational point of view the resulting line is asymmetric, and the signal is circumscribed to wavelengths longer than $\operatorname{Ly} \alpha$.

The detection of dim objects is a difficult task and very often the results may vary depending on subtle details. In the case of LAEs, the spectral asymmetry of the host galaxy continuum background with respect to the $\operatorname{Ly} \alpha$ line may enhance the detection of objects with the largest contribution of the continuum to the total signal. If we use a narrowband filter with an FWHM comparable to the EW of the Ly $\alpha$ line, the contribution of the continuum to the total recorded intensity may change significantly depending on the redshift of the object. Figure 1 shows an example of this effect on an ideal rectangular filter of a width of $132 \AA$, similar to the FWHM of the NB921 Subaru filter. The figure shows two simplified LAE spectra at slightly different redshifts (6.51 and 6.61). The objects have an observed FWHM of $10 \AA$ for the Ly $\alpha$ line and an identical continuum level. For the LAE at the lowest redshift, the Ly $\alpha$ line lies on the short-wavelength edge of the filter, and its EW is 2.5 times the wavelength range of the filter (i.e., $330 \AA$ in the observer's frame or $44 \AA$ in the rest-frame), which corresponds to a difference of one magnitude between the line and the continuum, a criterion often used to select LAE candidates in narrowband surveys (e.g., Ouchi et al. 2010; Kashikawa et al. 2011). For the LAE at the highest redshift, to reach the same signal through the rectangular filter, the intensity of the Ly $\alpha$ line (or its EW) should be approximately 34\% higher than the line intensity of the former object.

The bias introduced by the asymmetric continuum profile at both sides of the $\operatorname{Ly} \alpha$ line is more pronounced as the EW 


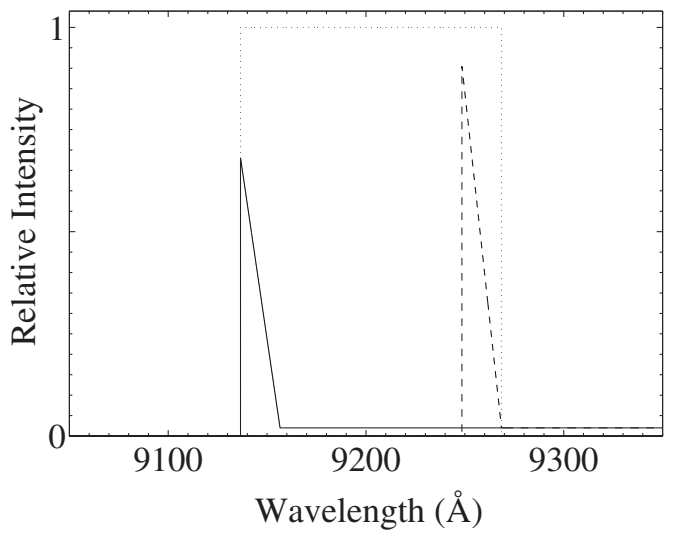

Figure 1. Example of the effect of the asymmetric continuum in the detection of LAEs. The amount of the continuum inside the ideal rectangular filter (dotted line) depends on the position of the Ly $\alpha$ break. In the extreme cases shown in this diagram, the continuum expands on the whole wavelength range of the filter for objects at the lowest redshift (solid line) or barely in the region covered by the Ly $\alpha$ line for objects at the highest redshift (dashed line). Both objects share the same continuum level, and the observed FWHM of the Ly $\alpha$ lines is $10 \AA$. For the object at the lower redshift, the contribution of the Ly $\alpha$ line is 2.5 times that of the continuum in the range of the filter. For detecting the highest redshift object with the same total signal, the Ly $\alpha$ line must be approximately $34 \%$ more intense than the line of the lower redshift object.

decreases. The observed-frame EWs for LAEs have values $\sim 100 \AA$, similar to a narrowband filter FWHM. Therefore, the asymmetric continuum may affect the shape of the LF of LAEs inferred from narrowband surveys. As objects are less luminous, the volume actually studied depends on the amount of continuum in the range of the filter.

\section{SAMPLE SIMULATION}

Shimasaku et al. (2006) and Ouchi et al. (2008) have analyzed the redshift distribution of LAEs in Subaru's narrowband filters using Monte Carlo mock samples of LAEs. For the purpose of comparing the characteristics of samples obtained with the OSIRIS-TF and other instruments, we have also built a simulated sample of LAEs. This sample was made by modeling the LAE spectra with a superposition of an asymmetric triangular profile for the Ly $\alpha$ line plus a continuum, which is a simplified version of the profile model by $\mathrm{Hu}$ et al. (2004). The Ly $\alpha$ line profile consists of a sawtooth with the steeper inclination at the line's blue side. At wavelengths shorter than the Ly $\alpha$ line, the continuum is zero, and it has some constant non-zero value at wavelengths equal to or larger than the Ly $\alpha$ line. Altogether, we have simulated the spectra of 5000 LAEs.

We have used the Schechter function to model the LF of LAEs at $z \simeq 6.5$ :

$$
\phi=\phi_{*}\left(\frac{L}{L_{*}}\right)^{\alpha} \exp \left(\frac{-L}{L_{*}}\right) \frac{d L}{L_{*}},
$$

with the parameters given by Kashikawa et al. (2011): $\alpha=$ $-1.5, \log \left(L_{*} / h^{-2} \operatorname{erg~s}^{-1}\right)=42.76$, and $\log \left(\phi_{*} / h^{3} \mathrm{Mpc}^{-3}\right)=$ -3.28 .

The simulated LF sample was computed through the inversion method of the cumulative Schechter function, integrating between $10^{41}$ and $10^{44} \mathrm{erg} \mathrm{s}^{-1}$, a luminosity range that broadly includes the observed LAEs at redshifts $z \approx 6.5$ (e.g., Kashikawa et al. 2011).
A random FWHM sample for the Ly $\alpha$ line has been computed according to the distribution parameters inferred from Table 2 in Kashikawa et al. (2011). These authors have measured the observed FWHM of $28 z \approx 6.5$ LAEs with values between $5.04 \AA$ and $25.2 \AA$, with mean of $13 \AA\left(428 \mathrm{~km} \mathrm{~s}^{-1}\right)$ and a standard deviation of $5 \AA$. The FWHM sample has been shifted to the rest frame of the LAEs to build the profile of the emitted Ly $\alpha$ line.

The random EW sample at rest frame $\left(\mathrm{EW}_{0}\right)$ has also been computed like the luminosity, using the inversion method on the cumulative function extracted from Figure 11 in Kashikawa et al. (2011). We tried to fit the EW distribution using exponential cumulative functions, but we obtained a better fit using the lognormal cumulative function. Lognormal EW distributions also fit the $\left[\mathrm{O}_{\mathrm{II}}\right]_{\lambda 3727} \mathrm{EWs}$ in the local $(z<0.2)$ universe (Blanton \& Lin 2000) and have been employed by Shimasaku et al. (2006) to characterize the EW of LAEs at redshift $(z \simeq 5.7)$. However, the $\mathrm{Ly} \alpha \mathrm{EW}$ distribution in LBGs at $z \sim 3$ does not show lognormal profile (Shapley et al. 2003), possibly because in LBGs Ly $\alpha$ is observed both in emission and in absorption, depending on the object. In any case, accurate EW measurements are difficult mainly because of the low level of the galaxy continuum, and thus the actual shape of the Ly $\alpha$ EW distribution is still an open subject. The $\mathrm{EW}_{0}$ in our simulations has a median value of $68 \AA$, slightly below the value of $74 \AA$ reported by Kashikawa et al. (2011). By construction, the lower values of the simulation were restricted to $\mathrm{EW}>0$. Extreme values are useful to test the instrumental response and to set constraints to the distribution of real objects. If the simulations show that the instrument reaches a certain parameter or combination of parameter values, but the corresponding objects have not been observed, it is evidence that there are few if any of them. On the other hand, if the simulations show that the instrument cannot detect objects with certain combinations of parameter values, it remains an open question whether these objects exist or not. In any case, because the simulation is based on empirical parameter distributions, extreme values are very improbable, and they cannot significantly alter the statistical results of this study. For example, in our simulation we find 69 objects with $\mathrm{EW}_{0}<8 \AA$ and 77 with $\mathrm{EW}_{0}>600 \AA$ from 2708 OSIRIS-TF recovered LAEs; of course, these numbers depend on the EW distribution adopted, which may be critical for the low EW regime.

To set thresholds for the EWs of LAEs is not a simple task. In some cases the continuum may be undetected, and the maximum threshold cannot be determined. In the case of the minimum threshold, Stark et al. (2010) have proposed that the EW at the rest frame should be $\mathrm{EW}_{0}>55 \AA$ for LAEs. More recently, these authors have proposed a value of $25 \AA$ (Stark et al. 2011), which approaches the widely accepted minimum threshold of $\mathrm{EW}_{0}>20 \AA$ A. The intensity of the Ly $\alpha$ line and its EW may depend on intrinsic properties of the LAEs or on geometrical attributes (see Section 1). Moreover, the adopted threshold is a figure that probably depends also on the observational limits adopted to separate candidates efficiently. Thus, Stark et al. (2011) note that the measured rest-frame EWs of the Ly $\alpha$ line for 13 spectroscopically confirmed LAEs range between $9.4 \AA$ and $350 \AA$. Therefore, we have conserved even the most extreme values for the $\mathrm{EW}$ in our simulations. Besides, these values may be useful to make extreme-case differences between the respective methodologies to detect $\mathrm{LAE}$ candidates.

For each simulated LAE, once the power of the Ly $\alpha$ line $\left(L_{\mathrm{Ly} \alpha}\right)$ and its $\mathrm{EW}_{0}$ have been assigned, it is possible to calculate 
the spectral power $\Phi_{c}$ of the continuum:

$$
\Phi_{c}=\frac{L_{\mathrm{Ly} \alpha}}{\mathrm{EW}_{0}} .
$$

This spectral power is used to compute the spectral shape of the continuum at wavelengths $\lambda \geqslant \lambda_{\mathrm{Ly} \alpha}$. For wavelengths $\lambda \leqslant \lambda_{\mathrm{Ly} \alpha}$, the continuum is assumed to be zero, as the optical depth for photons with wavelengths shorter than the Ly $\alpha$ line is large. Equation (2) is also used to build a continuum of reference for all the wavelengths of interest in our analysis, which will be used later to estimate a broadband continuum emission necessary for the criterion of detection.

The observed spectra for both, the Ly $\alpha$ line and the continuum, have been calculated from the corresponding spectral luminosities taking into account the redshift for each simulated object. These lines and continuum spectra have been added in order to obtain the observed flux, from which we will get the photometry later in our analysis. We have constructed another spectrum of constant intensity for each object, calculated from the corresponding continuum of reference.

\section{NARROWBAND FILTERS}

In this section we analyze the outcome produced by narrowband filters applied to our simulated sample. Gronwall et al. (2007) have noted the effect of a nonsquare bandpass in the definition of survey volume and flux calibration for large photometric selected samples of emission-line galaxies. For these authors, the volume of space sampled is a strong function of line strength: objects with bright line emission can be detected even if the Ly $\alpha$ line lies in the wings of the filter, but for weak Ly $\alpha$ sources the line must lie near the bandpass center to be noticeable. Furthermore, ignoring the actual position of the line in the bandpass prevents a precise estimate of the effective filter transmission in the line position, affecting the flux calibration. Being aware of these constraints, we consider two narrowband filter profiles: an ideal rectangular filter to study the effect of a general passband filter on the detection of LAEs, and a filter with a response similar to that of Subaru NB921 attached to the Suprime-Cam, to study the effect of a real-life filter profile.

Figure 2 shows the distribution of the simulated sample, the ideal and the NB921 Subaru filters, and the OSIRIS-TF (see Section 5). The simulations yield approximately 400 objects per wavelength bin (each bin with a width of $25 \AA$ ).

\subsection{Ideal Rectangular Filter}

The ideal rectangular filter is defined such that

$$
T_{\lambda}= \begin{cases}1 & \text { if } 9130 \leqslant \lambda \leqslant 9262 \AA, \\ 0 & \text { otherwise }\end{cases}
$$

where $T_{\lambda}$ is the transmission of the filter as a function of the wavelength $\lambda$. The bandwidth of the filter defined above is $132 \AA$, which equals the FWHM of the Subaru NB921 filter (see Section 4.2).

Simulated LAEs are recovered according to two criteria, one for source detection and the other for LAE candidate discrimination. The detection criterion is fulfilled when the irradiance in the narrowband filter is $\geqslant 5 \times 10^{-18} \mathrm{erg} \mathrm{s}^{-1} \mathrm{~cm}^{-2}$, which corresponds to the Subaru flux limit (Ouchi et al. 2010). The discriminant criterion is a contrast condition between the narrowband and the broadband fluxes. This criterion is in fact a simplified version of the Ouchi et al. (2010) color criterion, but is

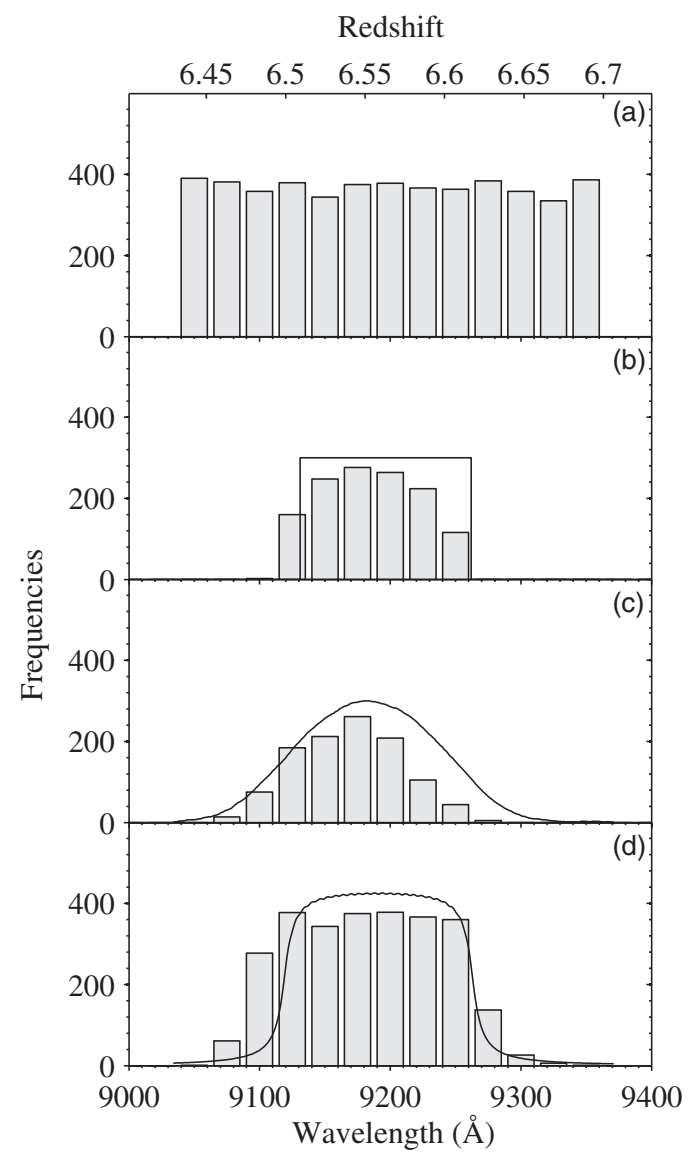

Figure 2. Number of simulations and detections. Panel (a) shows the absolute frequency per wavelength bin of the simulated population of LAEs. Panels (b) and (c) depict the number of detections computed for an ideal rectangular filter and for the Subaru NB921 filter; both panels show the transmission profile for the respective filter superposed. Panel (d) renders the number of detections computed for the OSIRIS-TF, along with the transmission profile of the wide filter (solid line) built using the band synthesis technique (see text).

sufficient to analyze the simulated data. In our case, the condition that must be accomplished consists of the narrowband flux being at least a magnitude brighter than the (adjacent) broadband flux, which provides a continuum of reference. Computationally, this criterion leads to the flux of the object measured through the filter being at least 2.5 times larger than the flux measured from the constant continuum of reference computed in Section 3.

Results are shown in Figure 2(b). Roughly, due to the asymmetric continuum bias, a larger number of detections at shorter wavelengths are expected, decaying smoothly at longer wavelengths. Small departures from this behavior are due to random deviations in the original simulated sample redshift distribution. Thus, most detections are scattered over the filter passband, but some simulated objects with their Ly $\alpha$ peaks lying at shorter wavelengths are recovered.

Only around one-fourth of the wavelength range in the leftmost bin in Figure 2(b) lies inside the filter profile, and almost half of the recovered objects in this bin correspond to simulations with the Ly $\alpha$ line peak located inside the filter passband. In fact, most recovered objects in this bin (83 out of 160) have the peak of the Ly $\alpha$ line at wavelengths shorter than the filter window, but with a fraction of the long-wavelength queue of this line inside the filter. Note that the detection of these objects will depend on the line parameters (peak position, FWHM, and intensity), 
and that the recorded signal will be diminished by the loss of the line flux outside the filter window, and thus these objects tend to have larger EWs that make detections easier. On the other hand, the smooth decay of the number of recovered LAEs along the filter passband is a result of the smaller quantities of the continuum emission lying inside the filter as the Ly $\alpha$ line peaks at longer wavelengths. For objects with their Ly $\alpha$ line near the long-wavelength extreme of the filter, the contribution of the continuum to the total flux in the band is negligible, and part of the long-wavelength queue of the line may also lie outside the filter window, yielding a steep drop in the number of detections. Therefore, these objects also tend to have large EWs to compensate for their total loss of flux.

\subsection{Subaru Filter}

The NB921 filter is characterized by an almost Gaussian profile with a central wavelength at $9196 \AA$ and an FWHM of $132 \AA$ (Kashikawa et al. 2011), which corresponds to a spectral resolution of 70 . The transmission profile is available from Subaru. ${ }^{9}$ A close inspection of this profile shows that the maximum of the recovered LAEs is slightly offset (9183 $\AA$ ) with respect to the filter peak, in agreement with the Ly $\alpha$ wavelength distribution of confirmed LAEs reported by Kashikawa et al. (2011).

We used the same detection criteria as in the case of the ideal rectangular filter. The results are shown in Figure 2(c). The number of recovered LAEs is restrained by the filter profile along with the same effects that have been noted in the ideal rectangular filter. On the one hand, there is the decay of the continuum contribution for the objects with the peak of the Ly $\alpha$ line closer to the long-wavelength limit of the filter; on the other hand, there also is the loss of the long-wavelength queue of the Ly $\alpha$ line, which spreads to the low transmission region of the filter profile. Therefore, the long-wavelength queue of the line observed through the Subaru filter is damped by the bell-shaped transmission, in agreement with Shimasaku et al. (2006) and Ouchi et al. (2008).

\section{TUNABLE FILTERS}

Throughout this work, a distinction is drawn between a frame, corresponding to one set of data read from the CCDs; an image, a number of frames at the same etalon settings which have been combined for analysis; and a field, a stack of images of the same area of sky at different etalon settings.

\subsection{OSIRIS Tunable Filters}

Basically, the imager/spectrograph OSIRIS-TF is a lowresolution Fabry-Pérot spectrograph which consists of a blue and a red arm. Our observations were performed with the red arm, which can be centered at any wavelength between 6500 and $9300 \AA$, and we observed using a fixed bandpass of $12 \AA$, which corresponds to a spectral resolution of about 770, a set of order-sorter filters to avoid contamination by neighboring orders, and a detector array consisting of two MAT $4 \mathrm{k} \times 2 \mathrm{k}$ CCDs (Cepa et al. 2003). The $12 \AA$ bandpass is the only one currently available for wavelengths around $9200 \AA$.

The observing strategy consists of sweeping a selected spectral range using steps of a half of the FWHM (in our case, 24 images shifted $6 \AA$ between 9122 and $9260 \AA$ ) to avoid aliasing. To apply this methodology to our simulated data, we

\footnotetext{
9 http://www.naoj.org/Observing/Instruments/SCam/sensitivity.html
}

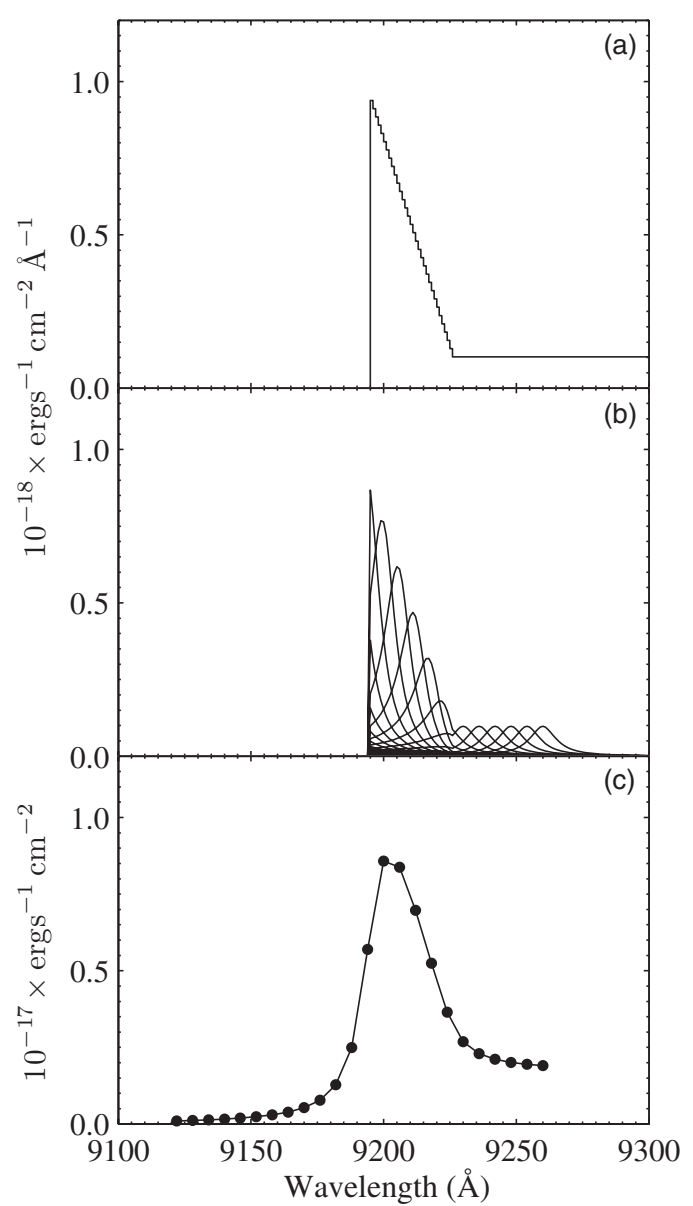

Figure 3. OSIRIS-TF output. Panel (a) shows the spectral flux density of an LAE at $z=6.6$ simulated spectrum. Panel (b) shows the set of OSIRIS-TF transmitted spectra for this simulation. Panel (c) renders the photometric output for each OSIRIS-TF, which is the signal integrated over each filter. Note that the units and the scale of the last plot are different from the two previous diagrams.

have modeled a set of 24, approximately Lorentzian-shaped, tunable filters using the following approximation that relates wavelengths and transmissions of the OSIRIS-TF (Cepa et al. 2011, Equation (3.14)):

$$
T \approx\left\{1+\left[\frac{2\left(\lambda-\lambda_{0}\right)}{\delta \lambda}\right]^{2}\right\}^{-1},
$$

where $\delta \lambda$ is the filter FWHM and $\lambda_{0}$ is the central wavelength.

Figure 3 shows a simulated LAE at redshift $z=6.6$ (a), the set of OSIRIS-TF filtered spectra for this simulation (b), and the photometric output (c). This particular simulation has a rather small $\mathrm{EW}_{0}=17.8 \AA$, which is adequate to make the continuum more apparent in the plot. In panel (c), the short-wavelength edge of the line aliases because the spectrum varies at a higher frequency than the OSIRIS-TF spectral resolution. Nonetheless, the asymmetry of the line profile is still noticeable.

In this paper we have adopted two criteria to retrieve LAEs from the simulations, based on the characteristics of OSIRIS-TF. The first is a detection condition that imposes an irradiance $\gtrsim 4 \times 10^{-18} \mathrm{erg} \mathrm{s}^{-1} \mathrm{~cm}^{-2}$ for the field narrowband image. This field is built through the sum of the 24 ultra-narrowband filters to provide a wider synthetic filter (band synthesis technique; Cepa 2009). The second condition consists of a ratio between the maximum and minimum of the 24 filters larger 
Table 1

High-redshift OSIRIS-TF Candidates

\begin{tabular}{|c|c|c|c|c|c|c|}
\hline \multirow[t]{2}{*}{$\mathrm{ID}^{\mathrm{a}}$} & \multirow{2}{*}{$\begin{array}{c}\text { R.A. }^{\text {b }} \\
\text { (hh:mm:ss.s) }\end{array}$} & \multirow{2}{*}{$\begin{array}{c}\text { Decl. } \\
\text { (dd:mm:ss) }\end{array}$} & \multirow[t]{2}{*}{ Redshift } & \multirow[t]{2}{*}{ Irradiance ${ }^{c}$} & \multicolumn{2}{|c|}{ Candidates } \\
\hline & & & & & OSIRIS-TF only & OSIRIS-TF and SED \\
\hline $\mathbb{A a}$ & $20: 56: 23.8$ & $-4: 40: 07$ & 6.494 & $4.01 \pm 0.09$ & LAE & Interloper/[O II $]$ emitter \\
\hline $\mathbb{A b}$ & $20: 56: 25.0$ & $-4: 37: 07$ & 6.498 & $1.05 \pm 0.09$ & LAE & $\mathrm{LBG}$ \\
\hline $\mathbb{A} \mathbf{c}$ & $20: 56: 23.8$ & $-4: 37: 02$ & $\lesssim 6.494$ & $1.12 \pm 0.09$ & LAE & Interloper/[O II $]$ emitter \\
\hline $\mathbb{B a}$ & $20: 56: 38.1$ & $-4: 40: 04$ & $\gtrsim 6.448$ & $1.49 \pm 0.09$ & $\mathrm{LAE} / \mathrm{LBG}$ & Interloper \\
\hline $\mathbb{B b}$ & $20: 56: 26.4$ & $-4: 37: 14$ & $\gtrsim 6.512$ & $1.67 \pm 0.09$ & $\mathrm{LAE} / \mathrm{LBG}$ & Interloper/[O II $]$ emitter \\
\hline $\mathbb{C a}$ & $20: 56: 16.7$ & $-4: 37: 53$ & 6.513 & $1.00 \pm 0.09$ & LBG & Young spiral galaxy \\
\hline $\mathbb{C b}$ & $20: 56: 30.6$ & $-4: 37: 37$ & 6.502 & $1.82 \pm 0.09$ & LBG & LBG \\
\hline
\end{tabular}

Notes.

${ }^{a}$ Candidate identifier.

b Epoch J2000.

${ }^{\mathrm{c}}$ In units of $10^{-17} \mathrm{erg} \mathrm{s}^{-1} \mathrm{~cm}^{-2}$.

than 2.5 (i.e., one magnitude). These conditions are equivalent to those imposed to the Subaru survey.

Results are shown in Figure 2(d). The distribution of the simulated LAEs recovered using OSIRIS-TF expands the full range of wavelengths between 9122 and $9260 \AA$. A visual inspection of Figures 2(a) and (d) shows that the shape of the distribution in this range of wavelengths closely resembles that of the simulated LAEs, without any apparent bias introduced by the filter profiles. Also notice that almost all the simulated objects in this range are recovered. In addition, there also are some retrieved LAEs with their Ly $\alpha$ line peak $\left(\lambda_{\text {Ly } \alpha}\right)$ outside the 9122-9260 A range, which is a consequence of the OSIRIS-TF Lorentzian profile. These objects tend to have large EWs; and those with $\lambda_{\mathrm{Ly} \alpha}>9260 \AA$ tend to be brighter, too.

\subsection{OSIRIS-TF Pilot Observations}

Photometry was carried out at the GTC using OSIRIS-TF with FWHM of $12 \AA$ at five contiguous wavelengths separated by steps of $6 \AA$, and a field of view free of adjacent orders of about 8 arcmin on each side. The observations were performed with central wavelengths $\lambda_{c}=$ $9122 \AA, 9128 \AA, 9134 \AA, 9140 \AA$, and $9146 \AA$. The observation run was done on 2010 September 8 . A binning of $2 \times 2$ was used in fast (standard) readout mode $(200 \mathrm{kHz})$, with three dithered exposures per wavelength of $210 \mathrm{~s}$ each, and separated by a triangular offset pattern of 10 arcsec to eliminate diametric ghosts during data reduction, each called a frame. The night was photometric and the seeing varied from 0.75 to 0.82 arcsec during the observation run.

Following the observation of the cluster in all the wavelengths, a standard star was observed with the same instrumental settings (but different exposure times) for flux calibration.

Standard IRAF procedures for bias subtraction were used on the data. Super-flats (a flat generated by averaging the sky from scientific images where sources have been masked) were created from and divided to the scientific frames due to the unevenly lit dome flats. Because of the TF's small bandpass and position-dependent wavelength, all observations contain sky $(\mathrm{OH})$ emission rings which were subtracted to all frames with the IRAF package TFred,${ }^{10}$ which estimates the sky background, including the sky rings which are several arcminutes in diameter. The three frame offsets of each wavelength were aligned and combined to generate an image with a total exposure time of

\footnotetext{
10 Written by D. H. Jones for the Taurus Tunable Filter, previously installed on
} the Anglo-Australian Telescope; http://www.aao.gov.au/local/www/jbh/ttf/ .
$630 \mathrm{~s}$; these were later convolved to the worst seeing of 0.82 arcsec.

Also, a field was created convolving to the worst seeing of 0.82 arcsec and combining all aligned images of different wavelengths (band synthesis technique). This field has a total integration time of $3150 \mathrm{~s}$ and yields a detection limit irradiance of $9 \times 10^{-18} \mathrm{erg} \mathrm{s}^{-1} \mathrm{~cm}^{-2}$ integrated over the full wavelength range (36 $\AA$ ) of the synthetic band. We used SExtractor (Bertin \& Arnouts 1996) to make a catalog of detected sources in the field. We excluded sources from the catalog that were too bright for high-redshift galaxies. Photometry on each monochromatic image was performed using an aperture of 1.5 arcsec using the positions gathered by SExtractor. Then we selected possible candidates based on the maximum versus minimum flux ratio, excluding all those sources with a ratio below 2.5 (i.e., one magnitude). The remaining objects were carefully inspected by eye to reject faint cosmic rays, ghost residuals, and source contamination by nearby companions or located too close to the edge of the image; the region around the gap between the detectors was particularly clumped with fake detections. Finally, we selected those candidates that showed a photometric profile similar to those expected for LAEs and LBGs with either the Ly $\alpha$ line or the Lyman break lying, at least partially, inside the observed wavelength range. Given the likely range of Ly $\alpha$ emission-line widths and the wavelength sampling, we expect to observe this line in more than three adjacent passbands.

Table 1 summarizes the data for the candidates. Column 1 identifies the OSIRIS-TF candidate. Columns 2 and 3 show the right ascension and declination coordinates, respectively. Column 4 shows the redshift obtained from the peak of the alleged Ly $\alpha$ line or Lyman break. Column 5 is the total irradiance corresponding to the field. Columns 6 and 7 present the classification of candidates obtained using the OSIRIS-TF data and the SED fitting to Subaru, Hubble Space Telescope (HST), and Spitzer photometrical data.

Figure 4 shows the OSIRIS-TF images for each LAE and LBG candidate. Each row in this figure corresponds to a different object located at the center of a guiding circle. Despite candidate $\mathbb{B}$ a being near the upper border of the clipped image, it does not affect our analysis. This is also the case for the candidate $\mathbb{C a}$, which is near the diffuse border defined by the dithered gap between the detectors. The first column of frames in Figure 4 shows the field exposure obtained by piling up all the individual images; the rest of the columns show the candidates observed at the wavelength identified in the heading row. 


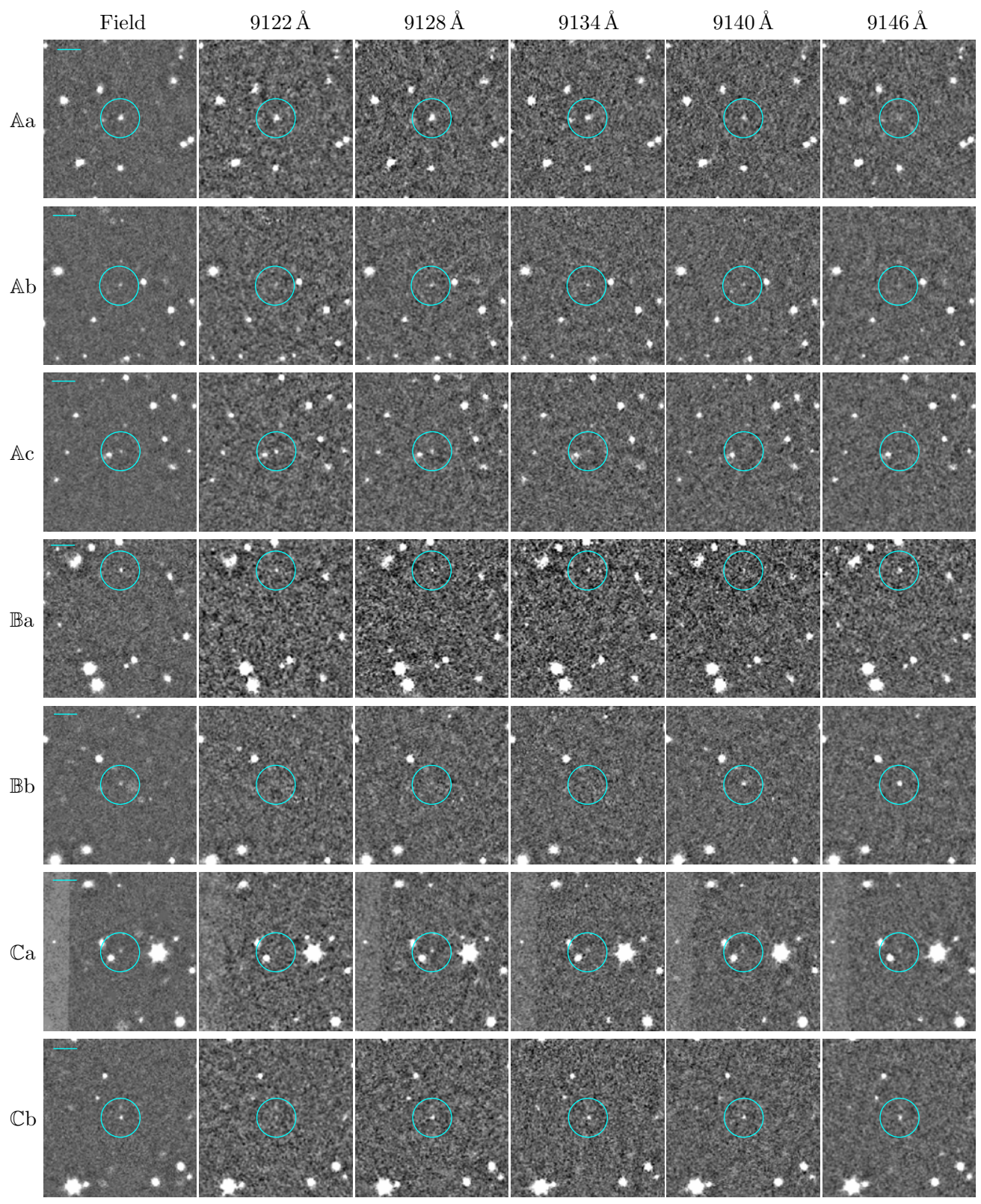

Figure 4. OSIRIS-TF observations. Column 1 shows the field obtained adding five individual images shown in Columns 2-6 labeled by the central wavelength of the tuned filter. The horizontal line at the top left of the fields marks a $6^{\prime \prime}$ scale. Each row corresponds to a different LAE or LBG candidate, identified by the labels at left. The objects lie in the center of the identifying circles.

(A color version of this figure is available in the online journal.)

Figure 5 shows three LAE candidates observed with the OSIRIS-TF at the GTC and selected simulations extracted from our database that resembles the real data, rather than a fitted model for each object. The shift in wavelengths between the observations and the model is due to the dependency of the tuned wavelength of the filter on the distance $r$ to the optical center in the OSIRIS-TF images (González et al. 2013): ${ }^{11}$

$$
\lambda=\lambda_{c}-5.04 r^{2}
$$

where $\lambda_{c}$ is the wavelength at the optical center expressed in $\AA$, and $r$ in arcminutes.

The redshifts for these LAE candidates were calculated identifying the brightest photometric point with the position

\footnotetext{
11 http://gtc-osiris.blogspot.com.es/
}

of the Ly $\alpha$ line. For the peak-missed LAE candidate shown in Figure 5(c), the redshift estimate is therefore an upper limit (see Table 1). In fact, the fraction of candidates with either upper or lower redshift limits is expected to be relatively high. This subject, and the possible contamination of the candidate sample by foreground galaxies, will be addressed in Sections 6 and 7 .

The two objects shown in Figure 6 have a photometric profile compatible with either LAE or LBG candidates. Their respective redshift lower limits are shown in Table 1 . The photometric profile for the object shown in Figure 6(a) may be contaminated by residuals of the sky-line subtractions at wavelengths below $9050 \AA$. As in the previous figure, simulated objects extracted from our database are also plotted for comparison purposes.

Objects shown in Figure 7 are two LBG candidates. Both objects show a steep increase in flux that is held at longer wavelengths, as expected for the continuum emission of LBGs. 
Table 2

Candidate Fluxes

\begin{tabular}{|c|c|c|c|c|c|c|c|}
\hline Filter & $\mathbb{A a}$ & $\mathbb{A b}$ & $\mathbb{A c}$ & $\mathbb{B a}$ & $\mathbb{B b}$ & $\mathbb{C a}$ & $\mathbb{C b}$ \\
\hline Suprime $V$ & $26 \pm 2$ & $<0.70$ & $4 \pm 1$ & $8.3 \pm 0.7$ & $\ldots$ & $36 \pm 1$ & $\ldots$ \\
\hline F606W & $\ldots$ & $\ldots$ & $\ldots$ & $\ldots$ & $\ldots$ & $41 \pm 7$ & $\ldots$ \\
\hline F702W & $\ldots$ & $\ldots$ & $1.5 \pm 0.2$ & $\ldots$ & $\ldots$ & $\ldots$ & $\ldots$ \\
\hline Suprime $i^{\prime}$ & $7.3 \pm 0.3$ & $7.5 \pm 0.2$ & $1.3 \pm 0.1$ & $11.0 \pm 0.3$ & $4.0 \pm 0.2$ & $\ldots$ & $9.7 \pm 0.2$ \\
\hline F814W & $\ldots$ & $\ldots$ & $0.5 \pm 0.2$ & $\ldots$ & $\ldots$ & $9.0 \pm 0.6$ & $\ldots$ \\
\hline OSIRIS-TF & $38 \pm 1$ & $18 \pm 2$ & $10 \pm 1$ & $22 \pm 2$ & $12.0 \pm 0.8$ & $15 \pm 1$ & $20.9 \pm 0-3$ \\
\hline $3.6 \mu \mathrm{m}$ & $0.55 \pm 0.03$ & $10.34 \pm 0.04$ & $<0.15$ & $0.41 \pm 0.06$ & $<0.15$ & $1.86 \pm 0.03$ & $0.44 \pm 0.02$ \\
\hline $4.5 \mu \mathrm{m}$ & $0.47 \pm 0.03$ & $6.37 \pm 0.03$ & $<0.08$ & $<0.08$ & $<0.08$ & $1.15 \pm 0.03$ & $0.24 \pm 0.03$ \\
\hline $5.8 \mu \mathrm{m}$ & $0.80 \pm 0.07$ & $3.47 \pm 0.07$ & $0.69 \pm 0.07$ & $<0.14$ & $<0.14$ & $0.77 \pm 0.07$ & $0.36 \pm 0.07$ \\
\hline $8.0 \mu \mathrm{m}$ & $<0.17$ & $1.73 \pm 0.08$ & $<0.17$ & $<0.17$ & $0.44 \pm 0.09$ & $<0.17$ & $<0.17$ \\
\hline
\end{tabular}

Note. All the fluxes in units of $10^{-19} \mathrm{erg} \mathrm{s}^{-1} \mathrm{~cm}^{-2} \AA^{-1}$.
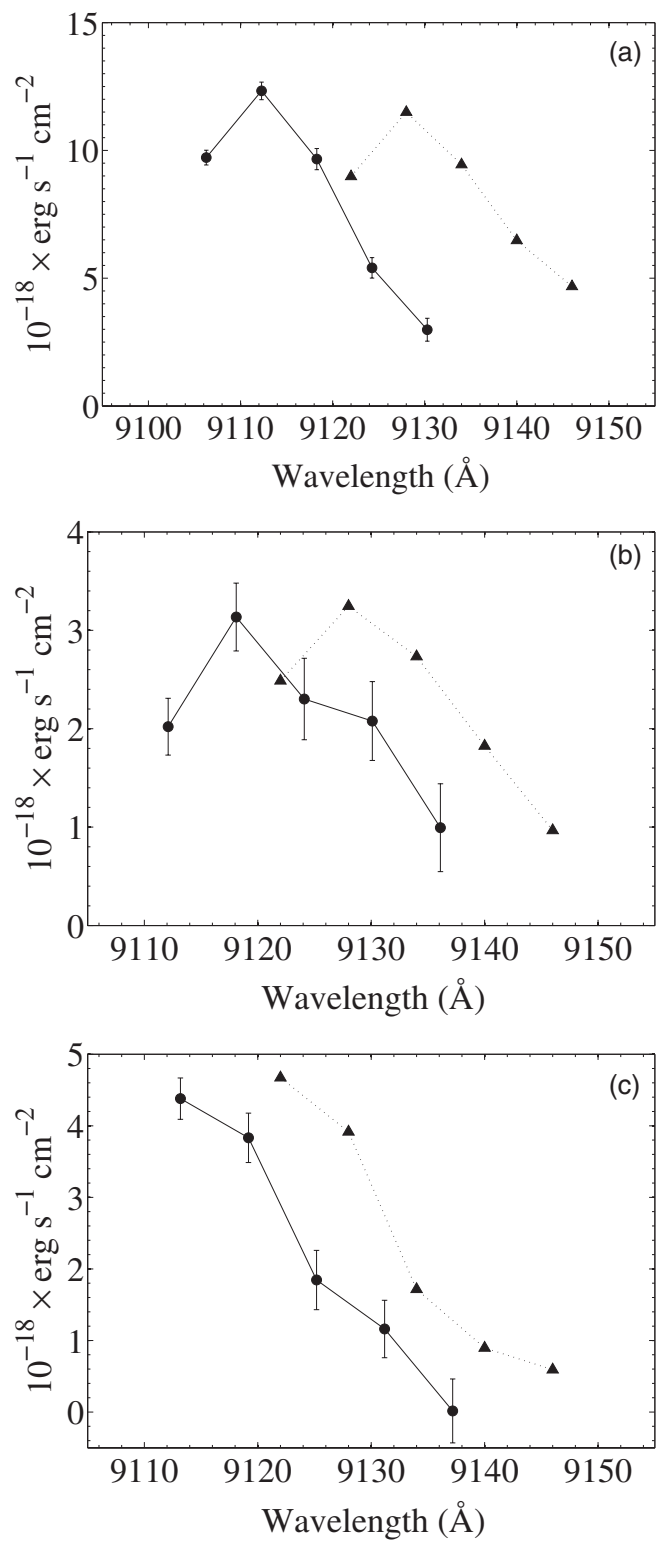

Figure 5. OSIRIS-TF LAE candidates. Panels (a), (b), and (c) correspond to candidates $\mathbb{A} a, \mathbb{b}$, and $\mathbb{A c}$, respectively. Circles connected by solid lines depict OSIRIS-TF photometrical data obtained at the GTC. Triangles linked by dotted lines show examples extracted from our simulations that resemble the real data. Photometrical data are shifted in wavelength with respect to the simulations due to the wavelength dependency of the OSIRIS-TF with the distance to the optical center.

\section{SUBARU, HST, AND SPITZER ARCHIVE DATA}

We have searched for additional photometric data for our LAE and LBG candidates in astronomical public databases and have found some deep images in the Subaru, HST, and Spitzer archives that cover, at least partially, the OSIRIS-TF field around MS 2053.7-0449. We used these data along with our observations to fit the SED of the OSIRIS-TF candidates, obtaining a more accurate classification (see Table 1). Table 2 shows the fluxes in the different bands of Subaru/Suprime-Cam, HST/WFPC2, and Spitzer/IRAC data. This table also includes the fluxes of the OSIRIS-TF band synthesis images, which correspond to a filter of FWHM $=36 \AA$. In the case of OSIRISTF fields and HST fluxes, we found discrepancies based on Subaru calibrations. We then used stars in the field to recalibrate the OSIRIS-TF and HST data to the Subaru photometry.

\subsection{Subaru Observations}

The Subaru/Suprime-Cam data were obtained from the Subaru-Mitaka Okayama-Kiso Archive System (SMOKA). They consist of data in filters $V, i^{\prime}$, and $z^{\prime}$ observed in 2009. The wavelength range of the $z^{\prime}$ filter includes our OSIRIS-TF observations, resulting in a helpful band to estimate the continuum. Data were reduced following the Suprime-Cam Data Reduction software (SDFRED2). The images were flat fielded, matched in point-spread function size for a predetermined target FWHM, scaled, and combined. The total integration time for $V$, $i^{\prime}$, and $z^{\prime}$ filters was 5040, 1920, and $1800 \mathrm{~s}$, respectively. The photometry for the candidates was performed with the qphot IRAF package, and fluxes were derived by measuring inside a 1.5 arcsec aperture.

OSIRIS-TF candidates $\mathbb{A a}, \mathbb{A b}, \mathbb{A c}$, and $\mathbb{B a}$ are seen in the $V$, $i^{\prime}$, and $z^{\prime}$ filters, pointing at possible interlopers (i.e., a galaxy at a lower redshift and with spectral features resembling those of LAEs over a limited range of wavelengths) or LBG candidates. Candidates $\mathbb{B b}, \mathbb{C a}$, and $\mathbb{C b}$ are not seen in at least one Subaru/ Suprime-Cam filter, but their best SED fit suggests they are all candidate interlopers.

\subsection{HST Observations}

The HST/WFPC2 data were obtained from the Multimission Archive at the Space Telescope Science Institute. They consist of two programs: ID5991 (filters F702W and F814W) and ID6745 (filters F606W and F814W). The proposals cover slightly different fields. Only two of the seven candidates fall in the images, namely, $\mathbb{A} c$ in the field of the proposal ID5991, and $\mathbb{C a}$ in the field covered by ID6745. 

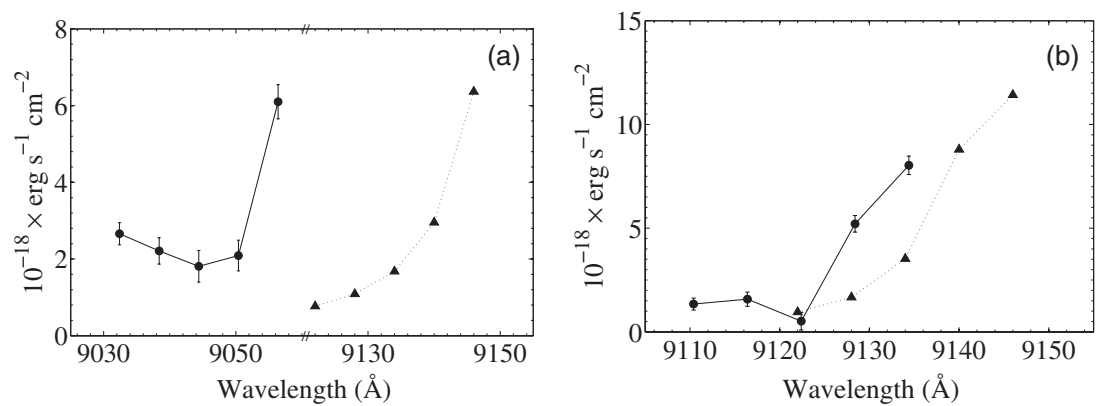

Figure 6. OSIRIS-TF LAE or LBG candidates. The same as in Figure 5, but the spectral profiles are compatible with both LAEs and LBGs. Panels (a) and (b) correspond to candidates $\mathbb{B a}$ and $\mathbb{B} b$, respectively.
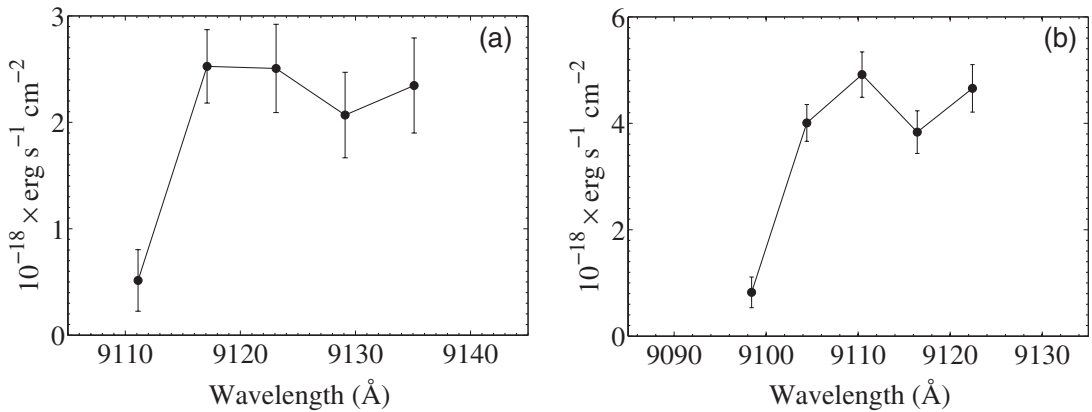

Figure 7. OSIRIS-TF LBG candidates. Panels (a) and (b) correspond to candidates $\mathbb{C a}$ and $\mathbb{C b}$, respectively. Circles with error bars linked by solid lines depict photometrical data obtained with the OSIRIS-TF at the GTC. The spectral profiles are compatible with LBGs.

The image reduction was performed using the IRAF/ STSDASS package. First, a warm-pixel rejection was applied to the images using the IRAF task warmpix. The cleaned images were then combined with the task crrej to remove cosmic-rays hits. Finally, the background was subtracted and the WFPC2 chips were combined using the task wmosaic. The total integration times for filters F702W and F814W were $2400 \mathrm{~s}$ and $2600 \mathrm{~s}$, respectively, for the proposal ID59991. For the proposal ID6745 the total integration times were $3300 \mathrm{~s}$ and $3200 \mathrm{~s}$ for filters F606W and F814W, respectively. The photometry for the seven candidates was performed in the HST filters with the IRAF package apphot. The magnitudes were derived by measuring fluxes inside a fixed circular aperture of 7 pixels $\left(\sim 0^{\prime} \cdot 7\right)$. OSIRIS-TF candidates found in these filters suggest an interloper nature.

\subsection{Spitzer/IRAC Observations}

A Spitzer/IRAC observation was gathered from the Spitzer Heritage Archive. The Astronomical Observation Request number 18626048 (program name/ID KTRAN-MS2053/30642; P.I. K.-V. Tran) was retrieved for this work. It is a four-channel $(3.6,4.5,5.8$, and $8 \mu \mathrm{m})$ IRAC map mode observation consisting of three rows and one column with a 42 point cycling dither pattern and "medium" scale (median separation between dither positions is 53 pixels; the IRAC pixel scale is approximately the same, $\sim 1.2$ arcsec in the four camera bands). The resulting map footprint is a roughly rectangular area of $22.9 \times$ 8.6 arcmin centered at $\alpha \approx 314.113, \delta \approx-4.670$. The major axis is oriented at P.A. $\approx 168.2$.

The data reduction of the four IRAC channels was performed using MOPEX v18.4.9, using as starting point the Corrected Basic Calibrated Data (CBCD). The IRAC CBCD data differ from the standard $\mathrm{BCD}$ products in the mitigation of several instrumental artifacts, including stray light, muxstripe, banding, muxbleed (electronic ghosting), column pulldown, and jail bars.
The Overlap and Mosaic pipelines were run to create mosaic images from the individual images. The former removes image background variations due to foreground light sources, while the latter removes defects and spurious pixels, reassembles the data onto a common pixel grid, and combines them into a mosaic with a corresponding noise map.

The four-channel photometry of the sources was performed by means of the Astronomical Point source EXtractor (APEX) tool provided within the MOPEX software. The User List Multiframe mode was used. In the APEX Multiframe mode, required for the IRAC instrument, the extraction is carried out simultaneously on the stack of input images rather than on a single mosaic image. The position and flux density estimate is provided for each detected source. The User List mode was chosen, providing APEX with an input list of object positions rather than using the Detect module to automatically find the sources in the field. Point-response function fitting and aperture photometry were performed on the input list objects. The centroids of the photometrical profiles were allowed to move slightly with respect to the input positions, resulting in small shifts between $0^{\prime \prime} .05$ and $0^{\prime \prime} 6$, except for $\mathbb{B b}\left(1^{\prime \prime} .3\right)$. Only detections with signal-to-noise ratio $>3$ have been considered.

\subsection{SEDs and Photometrical Redshifts}

We have used an updated version of HyperZ (Bolzonella et al. 2000) and synthetic spectral templates of galaxies (Bruzual A., \& Charlot 1993; Bruzual \& Charlot 2003) and quasars (Hatziminaoglou et al. 2000) to fit the SEDs of the OSIRIS-TF LAE and LBG candidates. These templates correspond to different types of starburst, spiral, irregular, and elliptical galaxies and quasars. For fitting the SEDs, we used the available Subaru, HST, and Spitzer data. We included upper limits to compute the SED. We had to provide the transmission 


\begin{tabular}{lccc}
\multicolumn{4}{c}{ Table 3 } \\
SED Fits \\
\hline \hline ID & \multicolumn{2}{c}{ Starburst } & {$[\text { O II }]^{\mathrm{b}}$} \\
\cline { 2 - 4 } & Redshift $^{\mathrm{a}}$ & $\chi^{2}$ & $\chi^{2}$ \\
\hline $\mathbb{A a}$ & 3.0 & 26.2 & 31.3 \\
$\mathbb{A b}$ & 5.4 & 36.3 & $\ldots$ \\
$\mathbb{A c}$ & 3.0 & 18.3 & 19.4 \\
$\mathbb{B a}$ & 5.4 & 33.1 & $\ldots$ \\
$\mathbb{B b}$ & 5.3 & 4.6 & 5.7 \\
$\mathbb{C a}$ & 2.4 & $10.1^{\mathrm{c}}$ & $\ldots$ \\
$\mathbb{C b}$ & 5.5 & 3.1 & $\ldots$ \\
\hline
\end{tabular}

Notes.

${ }^{\text {a }}$ SED fit done with Subaru, HST, and Spitzer data.

$\mathrm{b}$ [O II] candidates would be at $z \simeq 1.45$.

${ }^{c}$ The shown best SED $\chi^{2}$ fit is for a young spiral galaxy.

profiles of the Spitzer/IRAC filters, ${ }^{12}$ which are not included in the HyperZ filter database. We did not include OSIRIS-TF data because the synthetic templates do not have enough resolution to fit emission lines, which may dominate over the flux in the synthetic filter. With these fittings we check whether the SEDs are compatible with LAEs and LBGs. The results are summarized in Table 3. Prudence should be exercised when using HyperZ to fit the data: the number of photometric bands is rather small to obtain accurate fittings, and usually it is possible to fit the SED of various types of galaxies at different redshifts. Therefore, in our case SED fitting is useful to discard candidates, but provides a moderate support for object classification, and the fits must be regarded with caution. Below we present the results of fitting SEDs to the OSIRIS-TF LAE, LAE/LBG, and LBG candidates.

\subsubsection{OSIRIS-TF LAE Candidates}

Figure 8 shows the photometry and the SEDs of the OSIRIS-TF LAE candidates.

$\mathbb{A}$ a: Candidate $\mathbb{A}$ a is detected in the Subaru $V, i^{\prime}$, and $z^{\prime}$ filters; lies out of the field of the HST images; and is also found in Spitzer images at 3.6-5.8 $\mu \mathrm{m}$, but not in the $8 \mu \mathrm{m}$ band. Figure 8(a) presents the OSIRIS-TF, Subaru, and Spitzer data available for this object, and the SEDs for a starburst galaxy at $z=3.03$ and an [O II] interloper at $z=1.45$. The OSIRIS-TF photometric point shows an excess that may be ascribed to [O II] emission. Figure 8(a) also shows the optical high-resolution SED template for a starburst with $E(B-V)<0.1$ by Calzetti et al. (1994) and Kinney et al. (1996). ${ }^{13}$ This high-resolution template has been moved to the starburst galaxy redshift of $z=1.45$, and it has been scaled using a third-degree polynomial to the low-resolution starburst SED template fitted by HyperZ. The resulting optical SED does not show the actual strengths of the emission lines, but gives an idea of what their appearance should be. The $\chi^{2}$, in Table 3, is not very different for the [O II] emitter and starburst fits. The source is an interloper, and most probably an $[\mathrm{O} \mathrm{II}]$ emitter.

$\mathbb{A b}$ : Photometric data and SED fitting for candidate $\mathbb{A b}$ are shown in Figure 8(b). The object is detected in Subaru filters $V$, $i^{\prime}$, and $z^{\prime}$; it is located out of the field of the HST images and is found in all the Spitzer/IRAC filters.

\footnotetext{
12 Available at NASA/IPAC Infrared Science Archive: http://irsa.ipac. caltech.edu/data/SPITZER/docs/irac/calibrationfiles/spectralresponse/.

13 Templates available at

http://www.stsci.edu/hst/observatory/cdbs/cdbs_kc96.html.
}

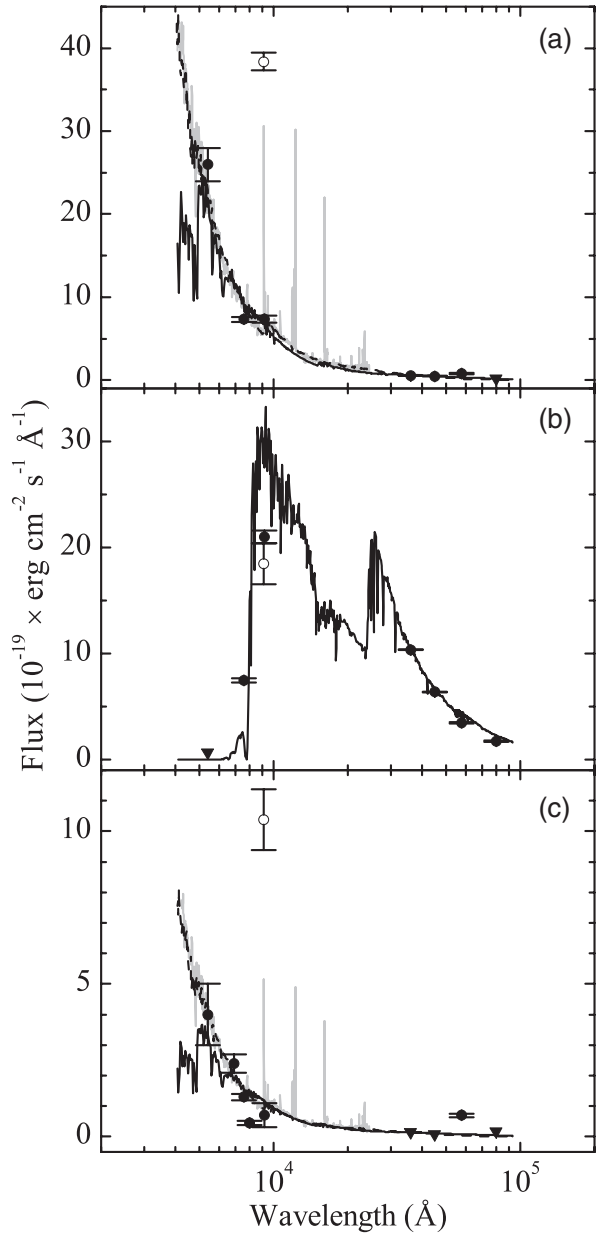

Figure 8. SED fittings for OSIRIS-TF LAE candidates. OSIRIS-TF (hollow circle), Subaru, HST, and Spitzer (filled circles for all three) fluxes are plotted along with SEDs calculated with HyperZ. (a) Candidate $\mathbb{A}$ a: the solid line corresponds to the best-fit SED, a $z=3.03$ starburst galaxy, the dashed line shows the fit at $z=1.45$ for an [O II] interloper, and the gray line shows a scaled high-resolution version of the previous fit, obtained from a starburst galaxy template by Calzetti et al. (1994) and Kinney et al. (1996). (b) Candidate $\mathbb{A}$ b: the best fit corresponds to a $z \simeq 5.4$ LBG. (c) Candidate $\mathbb{A c}$ : the best fit is obtained for a $z=3.0$ (solid line) starburst galaxy; dashed and gray lines show the fit and scaled templates for a $z=1.45[\mathrm{O}$ II] interloper, as for $\mathbb{A}$.

The photometric profile using only the OSIRIS-TF observations (see Figure 5(b)) suggests an LAE candidate. The profile of the best starburst SED fit at $z \simeq 5.4$ supports a candidate LBG, in which case the OSIRIS-TF photometry point probably corresponds to a spectral feature in the absorption area around the $9000 \AA$ wavelength. Therefore, object $\mathbb{A}$ b is classified as a possible LBG at $z \gtrsim 5.4$.

Ac: Figure 8(c) shows the photometric data for candidate $\mathbb{A c}$ and the SEDs of a starburst galaxy at $z=3.0$ and $z=1.45$. The object appears in all Subaru filters, in the field of the HST program ID5991, and is detected in the Spitzer $5.8 \mu \mathrm{m}$ image. Figure 8(c) is similar to Figure 8(a), with the gray line showing the optical high-resolution SED template for a starburst with $E(B-V)<0.1$.

The photometric profile of the OSIRIS-TF observations (see Figure 5(c)) resembles the long-wavelength queue of an emission line, but the HST, Subaru, and Spitzer data are not consistent with a $z \simeq 6.5 \mathrm{LAE}$ candidate. The fit for a starburst galaxy at $z=1.45$ matches the OSIRIS-TF photometric point corresponding to the $\left[\mathrm{O}_{\mathrm{II}}\right]_{\lambda \lambda 3726,3729}$ doublet. The $\chi^{2}$ values for 


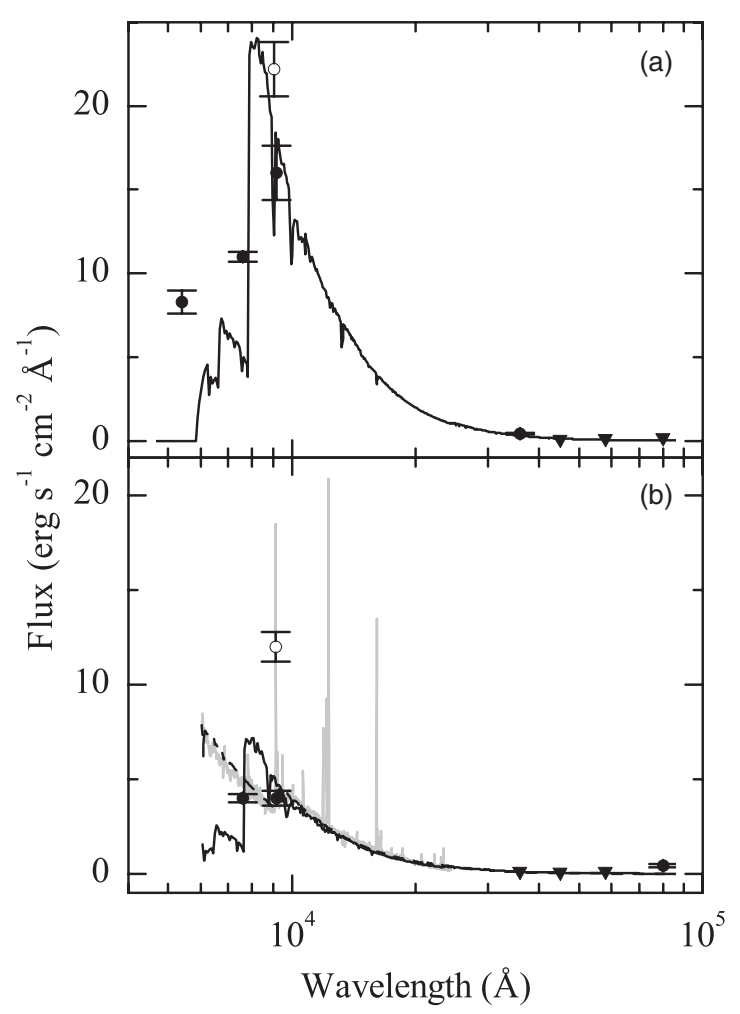

Figure 9. SED fittings for OSIRIS-TF LAE or LBG candidates. Fluxes obtained from OSIRIS-TF (hollow circle) and Subaru, HST, and Spitzer/IRAC (filled circles for the last three) images are plotted, along with SED fits calculated with HyperZ. (a) Candidate $\mathbb{B}$ a: the SED fit is for a starburst galaxy at redshift $z \simeq 5$.4. The object is most likely and interloper. (b) Candidate $\mathbb{B b}$ : photometric data have been fitted at $z \simeq 5.3$ (solid line) for a starburst galaxy, and at 1.45 (dashed line) for an [O II] emitter. The best fit suggests an [O II] interloper at the lowest redshift. The gray line is the same as in $\mathbb{A} a$ in Figure 8.

the best starburst fit and the [O II] interloper are comparable; altogether the object is likely the latter.

\subsubsection{OSIRIS-TF Double LAE/LBG Candidates}

Figure 9 shows the photometry and SED fitting for the OSIRIS-TF double LAE/LBG candidates.

$\mathbb{B a}$ : Candidate $\mathbb{B a}$ (shown in Figure $9(\mathrm{a})$ ) is detected in all of Subaru's filters, lies out of the field of the HST images, and is found in the Spitzer $3.6 \mu \mathrm{m}$ band. Figure 9(a) shows the photometric data available along with the best SED fit for a starburst galaxy at $z \simeq 5$.4. The flux in the OSIRIS-TF synthetic filter may be dominated by the short-wavelength queue of an emission line (Figure 6(a)), which explains why the OSIRIS-TF data are well above the Suprime-Cam $z^{\prime}$ band. The object is most likely an interloper.

$\mathbb{B b}$ : Figure 9(b) shows the photometric data for object $\mathbb{B b}$ and the SED of a starburst galaxy at $z \simeq 5.3$ and at 1.45 . The object is detected in the Subaru $i^{\prime}$ and $z^{\prime}$ filters, lies outside of the HST fields, and is also found in the Spitzer $8 \mu \mathrm{m}$ band. Figure 9(b) is similar to Figure 8(a), with the gray line showing the optical high-resolution SED template for a starburst with $E(B-V)<0.1$.

Note that, as mentioned earlier, the centroids of the photometrical profiles of the Spitzer/IRAC images for this object are shifted 1".3 with respect to their optical counterparts, and thus there is a possibility that the infrared images are misidentified. Both starburst galaxy SED fits are reasonable. However, the OSIRIS-TF flux is well above the Suprime-Cam $z^{\prime}$,

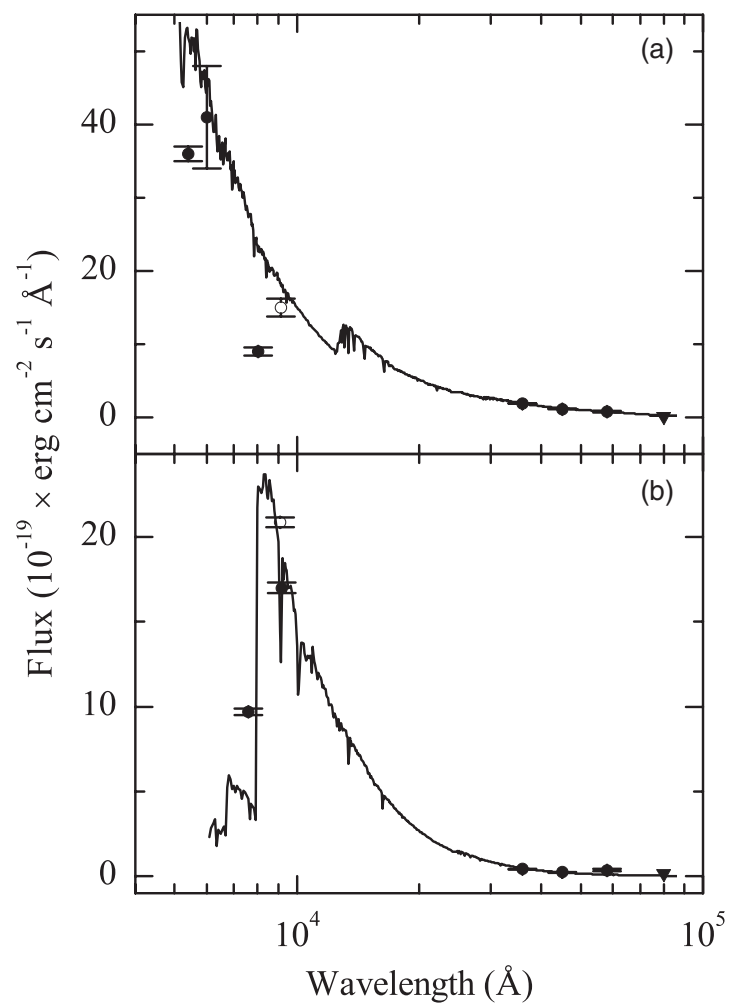

Figure 10. SED fittings for OSIRIS-TF LBG candidates. Fluxes obtained from OSIRIS-TF (hollow circles) and Subaru, HST, and Spitzer/IRAC (filled circles for the last three) images are plotted, along with SEDs calculated with HyperZ. (a) Candidate $\mathbb{C a}$ : the best SED fit is that of a spiral galaxy at redshift $z \simeq 2.4$. (b) Candidate $\mathbb{C b}$ : the photometric data are consistent with the SED of a starburst galaxy at $z=5.5$. The SED profile suggests that the object may be an LBG at $z \gtrsim 5.5$ or a high-redshift interloper.

suggesting that some of the short-wavelength wing of the [O II] line (see Figure 6(b)) lies in the OSIRIS-TF wavelength range. The object is probably an $[\mathrm{O} \mathrm{II}]$ interloper.

\subsubsection{OSIRIS-TF LBG Candidates}

Figure 10 shows the photometry and the SEDs of the OSIRIS-TF LBG candidates.

$\mathbb{C}$ a: Images for $\mathbb{C a}$ are available through the Subaru $V$ filter, HST program ID6745, and Spitzer 3.6-5.8 $\mu \mathrm{m}$ bands; it remains undetected in the $8 \mu \mathrm{m}$ observations and lies outside the field of Subaru's $i^{\prime}$ and $z^{\prime}$ filters. Figure 10(a) shows the photometric data for this object (see Figure 7(a) for the set of OSIRIS-TF observations). The OSIRIS-TF photometric point could correspond with an absorption line. We also plot the best fitting of an SED, which corresponds to a young spiral galaxy at $z \simeq 2.4$. The $H S T$ optical data are incongruent with any object at $z>4$. In any case, $\mathbb{C a}$ is likely a young spiral galaxy.

Cb: Figure 10(b) shows the photometric data and the SED of a starburst galaxy at $z=5.5$. The object is detected in Subaru's $i^{\prime}$ and $z^{\prime}$ filters; it lies out of the field of view of the HST images and is found in the Spitzer 3.6-5.8 $\mu \mathrm{m}$ filter but not in the $V$ and $8 \mu \mathrm{m}$ bands. The starburst SED fits the photometric data fairly well. The spectral profile observed with OSIRIS-TF (Figure 7(b)) may in fact be an artifact due to the absorption region at wavelengths longer than the Lyman break. Nonetheless, the SED fit sustains that $\mathbb{C b}$ is a reliable LBG candidate at $z \gtrsim 5.5$ rather than at 6.5 as estimated using only OSIRIS-TF data. 
Table 4

Simulation Statistics

\begin{tabular}{|c|c|c|c|c|c|}
\hline Instrument & Parameter $^{\mathrm{a}}$ & $N^{\mathrm{b}}$ & Median & $\mathrm{Q} 1^{\mathrm{c}}$ & $\mathrm{Q}^{\mathrm{d}}$ \\
\hline \multirow[t]{4}{*}{ IDEAL } & $z$ & 1290 & 6.55 & 6.53 & 6.58 \\
\hline & $L_{\mathrm{Ly} \alpha}{ }^{\mathrm{e}}$ & $\ldots$ & 6.35 & 4.16 & 10.93 \\
\hline & $\mathrm{EW}_{0}^{\mathrm{f}}$ & $\ldots$ & 94 & 56 & 175 \\
\hline & $\mathrm{FWHM}_{0}{ }^{\mathrm{f}}$ & $\ldots$ & 1.63 & 1.25 & 2.13 \\
\hline \multirow[t]{4}{*}{ Subaru } & $z$ & 1108 & 6.54 & 6.51 & 6.56 \\
\hline & $L_{\text {Ly } \alpha}{ }^{\mathrm{e}}$ & $\ldots$ & 7.49 & 4.85 & 13.23 \\
\hline & $\mathrm{EW}_{0}^{\mathrm{f}}$ & $\ldots$ & 105 & 66 & 187 \\
\hline & $\mathrm{FWHM}_{0}{ }^{\mathrm{f}}$ & $\ldots$ & 1.65 & 1.26 & 2.19 \\
\hline \multirow[t]{4}{*}{ OSIRIS-TF } & $z$ & 2708 & 6.55 & 6.51 & 6.59 \\
\hline & $L_{\text {Ly } \alpha}{ }^{\mathrm{e}}$ & $\ldots$ & 6.00 & 3.95 & 11.14 \\
\hline & $\mathrm{EW}_{0}^{\mathrm{f}}$ & $\ldots$ & 67 & 34 & 140 \\
\hline & $\mathrm{FWHM}_{0}{ }^{\mathrm{f}}$ & $\ldots$ & 1.67 & 1.27 & 2.16 \\
\hline
\end{tabular}

Notes.

a Modeled parameters studied (redshift, luminosity of the Ly $\alpha$ line, and the rest-frame EW and FWHM).

b Number of detections over the 5000 simulations.

${ }^{c}$ First quartile of the parameter distribution of the recovered LAEs.

d Idem for the third quartile.

e In units of $10^{43} \mathrm{erg} \mathrm{s}^{-1}$.

f In $\AA$.

\section{DISCUSSION}

In this section we compare the results obtained with the different instrumental models applied to our simulated LAE sample, and we examine different explanations for the results of the OSIRIS-TF pilot observations.

\subsection{Insights from Simulated Data}

Table 4 summarizes the modeled performance for detection of LAEs for the three filter models analyzed in this paper. We present the statistical results using non-parametric scores (sample median and quartiles) since most of the simulated variables are poorly fitted by the Gaussian distribution.

The main differences that can be drawn from Table 4 correspond to the number of detections and the EW distributions of the LAE recovered in each filter. The numbers of LAEs recovered from our simulation using the NB921 filter in the Subaru Suprime camera and the ideal rectangular filter are similar, but they are only about $40 \%$ of those selected using the OSIRIS-TF. These results are explained by the asymmetric continuum bias, the filter profiles, and the different power of narrowband and ultra-narrow-band survey methodologies to detect LAEs with small EW values.

It is difficult to detect small EW LAEs. Thus, it is very interesting that the distribution of the EWs at the rest frame for OSIRIS-TF-recovered LAEs shows a median $\left(\widetilde{E W}_{0}=67 \AA\right)$ that is very accurate with respect to the median of the simulations (68 $\AA$ ) and that is significantly smaller than those of the ideal and NB921 filters (94 and $105 \AA$, respectively). This result shows that the OSIRIS-TF can extend the search of LAE candidates to objects with a relatively low contrast between the line and the continuum fluxes, which otherwise would be underestimated or even unnoticed in narrowband surveys. Taking the values of the first quartile for the Subaru's EW in Table 4, we estimate that many LAEs with $\mathrm{EW}(\mathrm{Ly} \alpha)<60 \AA$ may remain undetected in Subaru's survey. This would explain the difference between the EW medians for LAEs at redshifts $z \simeq 5.7$ and 6.5 reported by Kashikawa et al. (2011;89 and $74 \AA$ A, respectively), as well as the lack of LAEs between redshifts $6.6<z<7.1$ with
$\mathrm{EW}(\mathrm{Ly} \alpha)$ in the range between 20 and $55 \AA$ accounted for by Pentericci et al. (2011). In fact, these changes in the EW of LAEs are interpreted as a fast evolution of the luminosity of the Ly $\alpha$ line caused by the incomplete reionization of the universe at redshifts $z>6$. If LAEs at $z \simeq 6.5$ and with $\mathrm{EW}(\mathrm{Ly} \alpha)<60 \AA$ were numerous, tunable filter surveys might have a large impact on our knowledge of their LF, changing our current view.

In addition to the EW, the mean luminosity of the Ly $\alpha$ line is also $5 \%$ and $20 \%$ lower for simulated LAEs detected with OSIRIS-TF with respect to those found using the ideal and Subaru filters, respectively. As we mentioned above, the OSIRIS-TF methodology to find LAE candidates presented in this paper is able to find almost all of our simulated objects. Now we see that OSIRIS-TF superior performance with respect to the other instruments is due not only to an unbiased wavelength coverage, but also to differences of the line luminosity properties of the detected LAEs.

The distribution of redshifts for the OSIRIS-TF candidates yields the largest interquartile range of $\Delta z=0.08$, in contrast to 0.05 for both the ideal and the NB921 filters. This difference between the OSIRIS-TF and the narrowband filters is a consequence of the respective filter transmission profiles. On one hand, narrowband filters tend to be effective in finding LAE candidates on a rather restricted range of wavelengths of the filter bandpass; on the other hand, OSIRIS-TF candidates are evenly distributed on the swept wavelength range between 9122 and $9160 \AA$. Moreover, the Lorentzian profile of the OSIRIS-TF transmission extends some filter sensitivity to find LAEs beyond the probed wavelengths. In practice, this will translate into an excess of LAE candidates with upper redshift limits and an excess of double LAE/LBG candidates with lower redshift limits at the blue and red borders of the set of OSIRIS$\mathrm{TF}$, respectively, as we have seen with the observed data presented in Section 5.2. Finally, the distributions of the FWHM at rest frame do not change significantly among the different filters.

The depth achieved using narrowband filters in LAE surveys is severely limited by the filter profile. Our results using simulations agree with the analysis of Subaru's data reported by Kashikawa et al. (2011). Thus, the output varies dramatically along the filter band, reflecting the filter response. In any case, the asymmetric continuum profile at each side of the Ly $\alpha$ line introduces a detection bias regardless of the profile of the narrowband filter. This bias is difficult to correct, as it may depend on several factors, such as the actual EW of the Ly $\alpha$ line. Therefore, narrowband surveys are useful to find candidates in a slim volume over a large area, but the properties that can be derived from follow-up spectroscopical observations are prone to produce biased results.

In contrast, the OSIRIS-TF can sample the whole range of the wavelengths of interest with a spectral resolution about 10 times larger than narrowband filters, and thus the combined Ly $\alpha$ line and break features could be recognized, and the redshift determined to a better accuracy (Figure 3 ). This avoids the biases introduced by the relatively large bandwidth and the extended wings of the transmission profiles of narrowband filters. LAEs with their Ly $\alpha$ line lying between 9122 and $9260 \AA$ are almost completely recovered (Figure 2). However, OSIRIS-TF also has limitations, in particular, the amount of total observing time increases with the number of images, which is proportional to the desired spectral resolution. Besides, the OSIRIS-TF relatively small field of view of $8.53 \times 8.67 \mathrm{arcmin}^{2}$, with a small shadowed area on one side, cannot compare to the 
Table 5

Dependence of Detections on the Number of Filters

\begin{tabular}{lccc}
\hline \hline $\begin{array}{l}\text { FWHM } \\
(\AA)\end{array}$ & $\begin{array}{c}\text { Number } \\
\text { of Filters }\end{array}$ & Detections & $\begin{array}{c}\mathrm{EW}_{0}{ }^{\mathrm{a}} \\
(\AA)\end{array}$ \\
\hline 12 & 24 & 2535 & $66 \pm 2$ \\
20 & 14 & 2542 & $66 \pm 2$ \\
30 & 9 & 2497 & $67 \pm 2$ \\
50 & 5 & 2282 & $72 \pm 2$ \\
60 & 4 & 1799 & $74 \pm 2$ \\
100 & 2 & 168 & $51 \pm 6$
\end{tabular}

Note. ${ }^{a}$ Median and error. The errors $(e)$ are computed from the interquartile $\operatorname{range}\left(r_{q}\right)$ by $e=0.7413 r_{q} N^{-1 / 2}$, where $N$ is the number of detections.

wide field of Subaru Suprime-Cam $\left(34 \times 27 \operatorname{arcmin}^{2}\right)$. As a low-resolution Fabry-Pérot spectrograph, the field of view of tunable filter instruments is limited by the dependence of the effective wavelength on the distance to the optical center (see Equation (5)), which would spoil the desired monochromaticity in wide-field images (although this effect can be compensated for by wavelength scanning at the expense of telescope time). Thus, OSIRIS-TF is an instrument suitable for pencil-beam surveys spanning a relatively large volume, and for assessing the biases produced by standard narrowband filter surveys.

We have looked for relationships between the simulated variables. Aside from the obvious dependences imposed by candidate selection criteria (e.g., detection and EW limits), there are no practical differences between the detected and non-detected sets of simulated data, regardless of the filter characteristics. The only tiny effects that we have found involve LAEs with the largest observed FWHM and located near any of the edges of the filter. Thus, objects near the blue edge, but with the Ly $\alpha$ peak off the filter range, may be still detected because part of the flux lies in the filter. On the other hand, objects near the filter red edge may be undetected because the long-wavelength queue of the Ly $\alpha$ line extends beyond the transmitted wavelengths.

We have also investigated the effect of the number of filters used to detect LAEs. This effect may be present in observational strategies using several narrow bands to detect LAE candidates (or in general any emission objects), such as the OSIRIS-TF procedure discussed in this paper. The particular filter profile may render small changes on the results, and thus we have used ideal filter sets (rather than Gaussian or Lorentzian profiles) to characterize the several sets with different numbers of filters. The filter passband is different for each set, thus they cover the same spectral region and effective volume. In any case, the separation between the centers of two adjacent filters is half of the FWHM of the filter passband, as in the case of the OSIRIS-TF.

Table 5 and Figure 11 summarize the results obtained with different sets of ideal filters. The wavelength range $(r)$ at the filters' FWHM covered for all the sets is the same, and it is given by the expression

$$
r=\frac{n+1}{2} w=150 \AA,
$$

where $n$ is the number of filters in the set and $w$ is the filter FWHM. We notice that the number of detections, around 2500 objects, decays slightly (3\%) from 24 to 9 filters. However, for five filters there is a sharp cutoff in this number, and for the set of two filters, only 168 are detected. For the line luminosity and

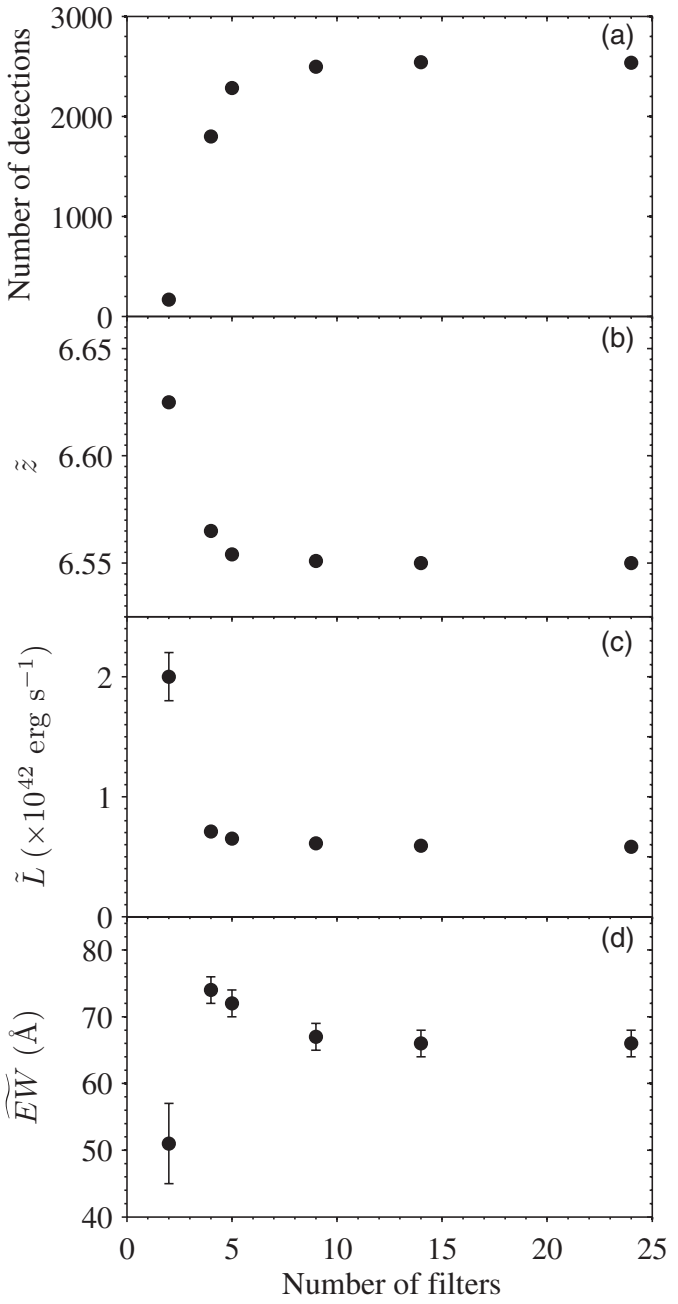

Figure 11. Dependences on the number of filters. This figure shows the results obtained using different sets of ideal (rectangular) filters on the LAE simulation data set. Each of these sets consists of a different number of filters, but they cover the same wavelength range as the original OSIRIS-TF set. Filters in a given set share the same FWHM, and their centers differ in steps of half this FWHM. For detections, we have adopted the same criteria as in the case of OSIRIS-TF. Panels show (a) the number of detections, (b) the median redshift, (c) the line luminosity, and (d) the rest-frame EW. The results for seven or more filters are similar, but for a smaller number of filters the number of detections falls dramatically, and the other parameters also change significantly.

redshift, the change also starts at five filters, with increments in their values. The rest-frame EW shows also an increment for five filters, but abruptly decays for two filter sets. The reason for this decay is that the only 168 objects detected are very luminous in both line and continuum emission. The rest-frame FWHM of the line, not shown in Figure 11, may also show an increment, but it is less significant than for the previous variables.

We note that for the two-filter set the distribution of detected objects becomes bimodal, rather than reflecting the original distribution. This occurs because a line located at the middle of the wavelength range lies in two filters, and our detection algorithm is unable to recognize such a line when the number of filters is small.

For Ly $\alpha$ and in general for other single and isolated emission lines, the previous analysis indicates that we might save significant telescope time, and still obtain similar results, observing through 9 filters with FWHM of $30 \AA$ rather than 24 filters with FWHM of $12 \AA$. In return, the lower spectral resolution may increase the number of interlopers. However, currently 
Table 6

OSIRIS-TF Survey Expected Quantities

\begin{tabular}{|c|c|c|}
\hline \multirow[t]{2}{*}{ Parameter } & \multicolumn{2}{|c|}{ Observations $^{\mathrm{a}}$} \\
\hline & Planned & Accomp \\
\hline Slices $^{\mathrm{b}}$ & 24 & 5 \\
\hline Irradiance limit ${ }^{\mathrm{c}}$ & 4 & 9 \\
\hline$L_{\mathrm{Ly} \alpha} \operatorname{limit}^{\mathrm{d}}$ & 2.1 & 4.7 \\
\hline Volume LAEs ${ }^{\mathrm{e}}$ & 8760 & 1503 \\
\hline Expected LAEs ${ }^{f}$ & 4.2 & 0.1 \\
\hline$L_{[\mathrm{O} \text { II }]} \operatorname{limit}^{\mathrm{g}}$ & 2.9 & 6.4 \\
\hline Volume $[\mathrm{O} \text { II }]^{\mathrm{e}}$ & 122 & 21 \\
\hline Expected [O II] $(\text { Takahashi })^{\mathrm{h}}$ & 7.4 & 0.8 \\
\hline Expected [O II] (Dressler) ${ }^{\mathrm{i}}$ & 2.5 & 0.2 \\
\hline
\end{tabular}

Notes.

a State of the observations: Planned or Accomplished.

b Number of wavelength slices.

${ }^{\mathrm{c}}$ Irradiance lower limit $\left(\times 10^{-18} \mathrm{erg} \mathrm{s}^{-1} \mathrm{~cm}^{-2}\right)$.

${ }^{\mathrm{d}}$ Ly $\alpha$ luminosity lower limit $\left(\times 10^{42} \mathrm{erg} \mathrm{s}^{-1}\right)$.

e Proper volume covered $\left(\mathrm{Mpc}^{-3}\right)$.

${ }^{\mathrm{f}}$ Number of expected LAEs obtained through the Schechter function with Kashikawa et al. (2011) parameters. Numbers are given with a precision of one decimal place, rather than integers, to ensure that at least one significant digit is shown.

g [O II] luminosity lower limit $\left(\times 10^{40} \mathrm{erg} \mathrm{s}^{-1}\right)$.

h Takahashi et al. (2007).

${ }^{\mathrm{i}}$ Dressler et al. (2011).

the OSIRIS-TF resolution cannot be changed in the wavelength range of our observations. It is worth noting that for other studies where line deblending is necessary, such as mapping $\mathrm{H} \alpha+[\mathrm{N}$ II] or the [S II] doublet, it may be convenient to maintain a filter bandpass lower than $15 \AA$ (e.g., Lara-López et al. 2010; Cedrés et al. 2013).

\subsection{Excess of Candidates and Interlopers}

The pilot observations obtained with the OSIRIS-TF embrace 5 out of 24 adjacent wavelength slices included in our complete program to find LAE candidates. Table 6 shows the values of some calculated and expected quantities.

Given the LAEs and [O II] interlopers LFs, we hardly expected to find any of these objects in the limited set of observations presented in this paper. Regardless of this prospect, we have extracted five candidates for which OSIRIS-TF data are congruent with LAEs, two of them also LBG candidates (in total, there were four LBG candidates). All of the LAE candidates were rejected after fitting broadband SEDs, but then three of them showed as possible [O II] interlopers, which is also a number of objects higher than expectations. These discrepancies between the number of expected LAEs and [O II] interlopers and the actual number of candidates are explained due to the low number of filters used in our current observations. Thus, the available data expand only a very small range of wavelengths (the FWHM of the synthetic OSIRIS-TF is $36 \AA$ rather than $150 \AA$ for the complete set of 24 filters), preventing a reliable sampling of the line and the continuum to both sides of the line. As a result, we cannot distinguish between small and large FWHM lines, because only a part of the wing of a line is observed, or even discriminate between emission lines and partially observed absorption features. These problems can be easily solved by observing through the complete set of filters. Meanwhile, we need to rely on archive data to improve candidate selection. Under these circumstances, even the limited
Table 7

Observer-frame FWHM for $z \simeq 6.5$ LAEs and Interlopers

\begin{tabular}{lccc}
\hline \hline Line & Redshift & $\begin{array}{c}\text { Velocity } \\
\left(\mathrm{km} \mathrm{s}^{-1}\right)\end{array}$ & $\begin{array}{c}\text { FWHM } \\
(\AA)\end{array}$ \\
\hline $\mathrm{Ly}_{\lambda 1216}$ & 6.50 & 400 & 12.16 \\
{$\left[\mathrm{O}_{\mathrm{II}}\right]_{\lambda \lambda 3726-9}$} & 1.45 & $\ldots$ & $\sim 10$ \\
{$[\mathrm{O} \text { III }]_{\lambda 5007}$} & 0.82 & 100 & 3.04 \\
$\mathrm{H} \alpha_{\lambda 6563}$ & 0.39 & 100 & 3.04 \\
\hline
\end{tabular}

number of broadband archive data is useful to reject candidates with SED profiles not compatible with LAEs or LBGs, but insufficient to confirm the nature of the objects.

Dressler et al. (2011) have calculated the LF for [O II], [O III], and $\mathrm{H} \alpha$ interlopers for LAEs at $z \simeq 5.7$. These LFs have a sharp cutoff for luminosities $\gtrsim 3 \times 10^{41} \mathrm{erg} \mathrm{s}^{-1}$, [O II] emitters being the most numerous of the foreground sources. Considering a similar cutoff for $z \simeq 6.5$ interlopers, it corresponds to irradiances $>2.2 \times 10^{-17} \mathrm{erg} \mathrm{s}^{-1} \mathrm{~cm}^{-2}$ for [O $\left.\mathrm{II}\right]$ at $z=1.45,>9.1 \times 10^{-17} \mathrm{erg} \mathrm{s}^{-1} \mathrm{~cm}^{-2}$ for [O III] at $z=0.82$, and $>5.6 \times 10^{-17} \mathrm{erg} \mathrm{s}^{-1} \mathrm{~cm}^{-2}$ for $\mathrm{H} \alpha$ at $z=0.39$. Our LAE candidates listed in Table 1 have irradiances below all these flux cutoff values, the only exception being $\mathbb{A} a$, which slightly exceeds the [O II] flux cutoff. Therefore, interlopers can enhance the number of fake candidates, in accordance with the results obtained when fitting the SEDs in Section 6.

Ly $\alpha$ observed lines at $z=6.5$ are rather wide, with FWHM around $10 \AA$. Interlopers' emission lines have observed widths that usually are well below this value. Table 7 shows the observed FWHM for Ly $\alpha$ and possible interlopers. For the interlopers, a fiducial velocity for the emission lines arising from star formation regions of $100 \mathrm{~km} \mathrm{~s}^{-1}$ has been chosen. Of course, the interlopers have redshifts $z \ll 6.5$, and thus lower Doppler broadening than $\operatorname{Ly} \alpha$. Then, all the single lines, but the unresolved [O II] blend, have observed FWHM easily distinguishable from the Ly $\alpha$ with the OSIRIS spectral resolution. In the case of the $[\mathrm{O}$ II] blend, the separation between the individual line peaks, rather than the velocities, dominates the observed FWHM.

Given the detection limit for these observations $(9 \times$ $10^{-18} \mathrm{erg} \mathrm{s}^{-1} \mathrm{~cm}^{-2}$ ), [O $\left.\mathrm{II}\right]$ interlopers at $z \simeq 1.45$ with line luminosities brighter than $L_{[\mathrm{OII}]}>1.166 \times 10^{41} \mathrm{erg} \mathrm{s}^{-1}$ will be detected. This yields a number of $0.2-0.8$ expected [O II] interlopers in our data, depending on the LF adopted (Dressler et al. 2011; Takahashi et al. 2007, respectively). Expected numbers for the full set of planned observations are shown in Table 6. From Dressler et al. (2011), we expect a final efficiency of about $2 / 3$ to find LAEs, i.e., 2 LAEs for every [O II] interloper, when the program is fulfilled. The OSIRIS Multi Object SpectrographMode, soon available at the GTC, could be used for follow-ups if necessary.

The line ratio $\left[\mathrm{O}_{\mathrm{II}}\right] \lambda 3726 /\left[\mathrm{O}_{\mathrm{II}}\right] \lambda 3729$ between the individual lines that conform to the [O II] blend feature depends on the electronic density $N_{\mathrm{e}}$. Extreme cases have values $\lim _{N_{\mathrm{e}} \rightarrow 0}=1.5$ and $\lim _{N_{\mathrm{e}} \rightarrow \infty}=0.35$, and thus this line ratio can be used to calculate the electronic density when it is in the range $2<\log \left(N_{\mathrm{e}}\right)<4$ (Pradhan et al. 2006). Different values of [O II] ratios have a direct incidence on the unresolved blend FWHM measured with OSIRIS-TF, which makes it even more difficult to distinguish between LAEs and [O II] interlopers. There are few studies on the LF of [O II], and all of them deal with the blend as a single feature (Hogg et al. 1998; Gallego et al. 2002; Teplitz et al. 2003; Takahashi et al. 2007; Dressler et al. 2011). 
An effect to take into account is the distortion of the LF of the observed LAEs and [O II] interlopers, and thus their number counts, due to the redshift-dependent magnification bias (e.g., Bartelmann 2010; de Diego et al. 2011). This effect increases the number of observed faint sources, but reduces their number density, enlarging the angular distance between the sources. The overall result depends on the steepness of the number-count function. For the high-luminosity LAEs, such as those observed at $z \sim 6.5$, this function is steep and more sources become observable. The situation is more complex for [O II] interlopers, for which the high-luminosity objects do not dominate the number counts. Thus, the magnification bias may reduce the number of observed [O II] interlopers with luminosities below $\simeq 3 \times 10^{41} \mathrm{erg} \mathrm{s}^{-1}$ and enhances the counts for more luminous sources. In our case, the field observed with the OSIRIS-TF is dominated by the cluster of galaxies MS 2053.7-0449 at redshift $z=0.583$, which expands about $4 \times 7 \operatorname{arcmin}^{2}$. The cluster contains a gravitational lensed arc (Luppino \& Gioia 1992; Tran et al. 2005). The strong gravitational lens model has been discussed by Verdugo et al. (2007), and the weak-lensing signature of the cluster was detected by Hoekstra et al. (2002), who estimated a cluster velocity dispersion of $886 \mathrm{~km} \mathrm{~s}^{-1}$.

Following Hildebrandt et al. (2011), we have used the singular isothermal sphere approximation to calculate the lens magnification as a function of the angular separation $\theta$ from the cluster center:

$$
\mu(\theta)=\frac{\theta}{\theta-\theta_{E}},
$$

where $\theta_{E}$ is the Einstein radius (Narayan \& Bartelmann 1996) for the cluster:

$$
\theta_{E}=4 \pi\left(\frac{\sigma_{v}}{c}\right)^{2} \frac{D_{d s}}{D_{s}}
$$

and where $\sigma_{v}$ is the one-dimensional velocity dispersion (we have adopted Hoekstra et al. 2002, estimate of $886 \mathrm{~km} \mathrm{~s}^{-1}$ ), $D_{d s}$ is the angular diameter distance from the lensing cluster to the source, and $D_{s}$ is the angular diameter distance from the observer to the source. We have taken a common redshift of $z=6.5$ for LAEs and $z=1.45$ for [O II] interlopers. In our case, the Einstein radius for MS 2053.7-0449 is $\theta_{E}=17^{\prime \prime}$ and $11^{\prime \prime}$ for LAEs and [O II] interlopers, respectively.

The mean magnification $\langle\mu\rangle_{\mathrm{wl}}$ in the weak lensing regime of the OSIRIS-TF field around MS 2053.7-0449 can be calculated considering an angular separation $3 \theta_{E} \leqslant \theta \leqslant 4^{\prime}$ which ranges from the weak lensing limit to the edge of the detector:

$$
\langle\mu\rangle_{\mathrm{wl}}=\frac{\int_{3 \theta_{E}}^{4^{\prime}} \mu(\theta) \mathrm{d} \theta}{\int_{3 \theta_{E}}^{4^{\prime}} \mathrm{d} \theta},
$$

yielding mean magnifications of 1.17 for LAEs and 1.13 for [O II] interlopers. Figure 12 shows the change of the magnification with the distance to the cluster center. For the planned sample, this magnification yields an increase from 4.2 to 5.7 for expected LAE counts. However, for the currently observed bands, the expected number of LAEs has a negligible increase of about 0.1 objects. As discussed above, the case of [O II] interlopers is more complicated and depends on the actual profile of their LF.

A major concern with photometric searches of LAE candidates is that only a tight range of redshifts is probed, yielding small samples prone to cosmic variance due to large-scale density fluctuations. Cosmic variance accounts for deviations from

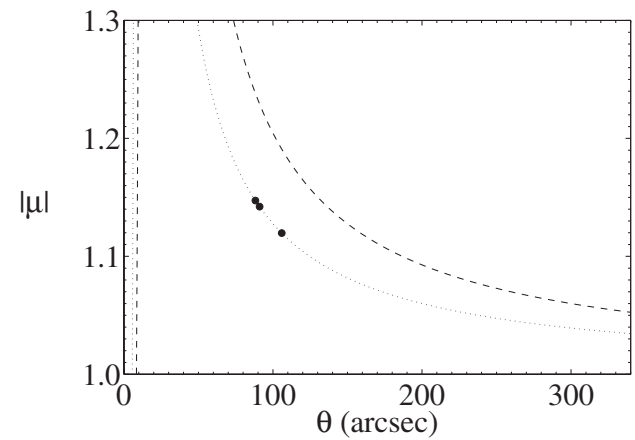

Figure 12. Magnification as a function of the angular separation to the center of the cluster of galaxies. The dotted line shows the absolute value of the magnification $\mu$ as a function of the angular separation $\theta$ for the cluster MS 2053.7-0449, using a singular isothermal sphere model. Filled circles correspond to [O II] interloper candidates.

the factual or expected values of the number counts of rare phenomena and of objects found in small volume surveys. Following Trenti \& Stiavelli (2008), we have calculated that the uncertainty on the number counts is $\sigma_{\text {counts }}=1$ for both LAEs and [O II] interlopers. Moreover, [O II] emitters are likely to show strong cross-correlation positions (Dressler et al. 2011). Therefore, we do not discard the possibility that some interloper candidates are actually [O II] emitters.

\section{CONCLUSIONS}

Narrowband surveys allow us to sample large sky areas and have been successful in finding LAE candidates. However, we have shown that the asymmetrical profile of the continuum around the Ly $\alpha$ line in LAEs yields a detection bias that affects the ability to find objects at redshifts where the Ly $\alpha$ line lies near the long-wavelength edge of narrowband photometric filters. Therefore, the Subaru survey and others that might be conducted using narrowband filters are prone to yield a biased LF. Besides, this methodology tends to ignore or underestimate small EW objects.

In the case of ultra-narrow-band surveys, our simulations show that the overall performance for LAE detection using OSIRIS-TF is not affected by the filter transmission profile, and the LF of LAEs could be accurately calculated. Moreover, OSIRIS-TF can recover simulated LAEs with Ly $\alpha$ line EWs significantly smaller than the objects recovered with narrowband filters. Nonetheless, tunable filters do not produce large monochromatic images, and the number of ultra-narrow-band images increases in proportion to the required spectral resolution, thus the size of the studied area is limited for practical reasons. Therefore, both narrowband and ultra-narrow-band surveys have different strengths and weaknesses, and thus they must be regarded as complementary strategies to study high-redshift LAEs.

We are carrying on a program to find LAE candidates with the OSIRIS-TF at the GTC. Part of this program is devoted to finding candidates at redshift $z \simeq 6.5$, with a strategy based on our study with Monte Carlo data. We have already performed pilot observations of five sets of images with the GTC and the OSIRIS-TF instrument at adjacent wavelengths, which is a fraction of the 24 wavelength slices that we plan to observe. The OSIRIS-TF images are separated by wavelength steps of $6 \AA$ and have a bandpass of $12 \AA$, covering a wavelength range of about $36 \AA$. The total exposure time in each wavelength is $630 \mathrm{~s}$, rendering a detection level of $9 \times 10^{-18} \mathrm{erg} \mathrm{s}^{-1} \mathrm{~cm}^{-2}$, which is about two times less sensitive than that reported 
for the Subaru LAE surveys. Available Subaru, HST/WFPC2, and Spitzer/IRAC archive data have been employed to build SED models. Because of the limited wavelength range of OSIRIS-TF observations, these models have been very helpful to reject $\mathrm{LAE}$ and $\mathrm{LBG}$ candidates and to identify interlopers, although the low number of bands used in the fits prevents accurate object classification and redshift estimate.

We have calculated the number of expected LAEs in the OSIRIS-TF field with our observational conditions. For this purpose, we have taken into account the weak lensing effect introduced by a nearby cluster of galaxies and the effect of the cosmic variance. Thus, we expected no more than one possible LAE and [O II] interloper showing up in our data. Actually, we have identified three possible [O II] interlopers and one LBG candidate. The possible overabundance of [O II] interlopers in the field might be a result of cross-correlation positions (Dressler et al. 2011). In any case, these results support the capabilities of OSIRIS-TF to perform an accurate survey of emission-line objects.

We plan to complete the set of OSIRIS-TF 24 wavelength slices to extract a sample of high-redshift candidates, namely, LAEs, LBGs, and high-redshift interlopers. The sample will be studied to confirm the nature of the objects using OSIRIS Multi-Object Spectrograph when available.

This research has been partially funded by the UNAMDGAPA-PAPIIT IN110013 Program. J.A.D. and M.A.D. are grateful for support from CONACyT grant CB-128556. J.A.D. is grateful for support from grant SAB2010-0011 awarded by the Spanish MIED through the "Programa Nacional de Movilidad de Recursos Humanos" included in the Plan Nacional de I-D+i 2008-2011. T.V. acknowledges support from CONACYT grant 165365 through the program "Estancias posdoctorales y sabáticas al extranjero para la consolidación de grupos de investigación." This work has been partially funded by the Spanish Ministry of Science and Innovation (MICINN) under the Consolider-Ingenio 2010 Program grant CSD2006-00070: First Science with the GTC (http://www.iac.es/consolider-ingenio-gtc), AYA201129517-C03-01, and AYA2011-29517-C03-02. Observations presented in this paper were made with the Gran Telescopio Canarias (GTC), installed in the Spanish Observatorio del Roque de los Muchachos of the Instituto de Astrofísica de Canarias, on the island of La Palma. The authors are thankful to the anonymous referee for the critical and constructive suggestions.

Facility: GTC (OSIRIS)

\section{REFERENCES}

Atek, H., Kunth, D., Hayes, M., Östlin, G., \& Mas-Hesse, J. M. 2008, A\&A, 488, 491

Atek, H., Kunth, D., Schaerer, D., et al. 2009, A\&A, 506, L1

Bartelmann, M. 2010, Gravitational Lensing, arXiv:1010.3829

Bertin, E., \& Arnouts, S. 1996, A\&AS, 117, 393

Blanc, G. A., Adams, J. J., Gebhardt, K., et al. 2011, ApJ, 736, 31

Blanton, M., \& Lin, H. 2000, ApJL, 543, L125

Bolzonella, M., Miralles, J.-M., \& Pelló, R. 2000, A\&A, 363, 476

Bruzual, G., \& Charlot, S. 2003, MNRAS, 344, 1000

Bruzual, A. G., \& Charlot, S. 1993, ApJ, 405, 538

Calzetti, D., Kinney, A. L., \& Storchi-Bergmann, T. 1994, ApJ, 429, 582

Cedrés, B., Beckman, J. E., Bongiovanni, Á., et al. 2013, ApJL, 765, L24

Cepa, J. 2009, TF User Manual, Tech. rep., Instituto de Astrofísica de Canarias Cepa, J., Aguiar, M., Bland-Hawthorn, J., et al. 2003, RMxAC, 16, 13

Cepa, J., Alfaro, E., Bongiovanni, A., et al. 2011, OSIRIS: User Manual (Scientific Use), Tech. rep., Instituto de Astrofísica de Canarias

Ciardullo, R., Gronwall, C., Wolf, C., et al. 2012, ApJ, 744, 110

Cowie, L. L., \& Hu, E. M. 1998, AJ, 115, 1319

Dayal, P., \& Ferrara, A. 2012, MNRAS, 421, 2568 de Diego, J. A., Cepa, J., De Leo, M., \& Bongiovanni, Á. 2011, JPhCS, 314, 012119

Dijkstra, M., Lidz, A., \& Wyithe, J. S. B. 2007, MNRAS, 377, 1175

Dressler, A., Martin, C. L., Henry, A., Sawicki, M., \& McCarthy, P. 2011, ApJ, 740,71

Finkelstein, S. L., Cohen, S. H., Moustakas, J., et al. 2011, ApJ, 733, 117

Finkelstein, S. L., Rhoads, J. E., Malhotra, S., Grogin, N., \& Wang, J. 2008, ApJ, 678,655

Forero-Romero, J. E., Yepes, G., Gottlöber, S., \& Prada, F. 2012, MNRAS, 419,952

Gallego, J., García-Dabó, C. E., Zamorano, J., Aragn-Salamanca, A., \& Rego, M. 2002, ApJL, 570, L1

González, J. J., Cepa, J., González-Serrano, I., et al. 2013, OSIRIS/GTC Red Tunable Filter: Wavelength Variations across the Field of View. The Anomalous Phase Effect, in press

Gronwall, C., Ciardullo, R., Hickey, T., et al. 2007, ApJ, 667, 79

Hansen, M., \& Oh, S. P. 2006, MNRAS, 367, 979

Hatziminaoglou, E., Mathez, G., \& Pelló, R. 2000, A\&A, 359, 9

Hayes, M., \& Östlin, G. 2006, A\&A, 460, 681

Hayes, M., Östlin, G., Schaerer, D., et al. 2010, Natur, 464, 562

Hibon, P., Kashikawa, N., Willott, C., Iye, M., \& Shibuya, T. 2012, ApJ, 744,89

Hildebrandt, H., Muzzin, A., Erben, T., et al. 2011, ApJL, 733, L30

Hoekstra, H., Franx, M., Kuijken, K., \& van Dokkum, P. G. 2002, MNRAS, 333,911

Hogg, D. W., Cohen, J. G., Blandford, R., \& Pahre, M. A. 1998, ApJ, 504, 622

Hu, E. M., \& Cowie, L. L. 2006, Natur, 440, 1145

Hu, E. M., Cowie, L. L., Capak, P., et al. 2004, AJ, 127, 563

Kashikawa, N., Kitayama, T., Doi, M., et al. 2007, ApJ, 663, 765

Kashikawa, N., Shimasaku, K., Malkan, M. A., et al. 2006, ApJ, 648, 7

Kashikawa, N., Shimasaku, K., Matsuda, Y., et al. 2011, ApJ, 734, 119

Kinney, A. L., Calzetti, D., Bohlin, R. C., et al. 1996, ApJ, 467, 38

Krug, H. B., Veilleux, S., Tilvi, V., et al. 2012, ApJ, 745, 122

Kunth, D., Mas Hesse, J. M., Terlevich, E., et al. 1998, A\&A, 334, 11

Lara-López, M. A., Cepa, J., Castañeda, H., et al. 2010, PASP, 122, 1495

Luppino, G. A., \& Gioia, I. M. 1992, A\&A, 265, L9

Malhotra, S., \& Rhoads, J. E. 2004, ApJL, 617, L5

Mallery, R. P., Mobasher, B., Capak, P., et al. 2012, ApJ, 760, 128

Narayan, R., \& Bartelmann, M. 1996, Lectures on Gravitational Lensing, arXiv:astro-ph/9606001

Neufeld, D. A. 1991, ApJL, 370, L85

Ono, Y., Ouchi, M., Mobasher, B., et al. 2012, ApJ, 744, 83

Ono, Y., Ouchi, M., Shimasaku, K., et al. 2010, ApJ, 724, 1524

Ota, K., \& Iye, M. 2012, MNRAS, 423, 444

Ota, K., Iye, M., Kashikawa, N., et al. 2010, ApJ, 722, 803

Ouchi, M., Shimasaku, K., Akiyama, M., et al. 2008, ApJS, 176, 301

Ouchi, M., Shimasaku, K., Furusawa, H., et al. 2010, ApJ, 723, 869

Pentericci, L., Fontana, A., Vanzella, E., et al. 2011, ApJ, 743, 132

Pradhan, A. K., Montenegro, M., Nahar, S. N., \& Eissner, W. 2006, MNRAS, 366, L6

Santos, M. R., Ellis, R. S., Kneib, J.-P., Richard, J., \& Kuijken, K. 2004, ApJ, 606,683

Schenker, M. A., Stark, D. P., Ellis, R. S., et al. 2012, ApJ, 744, 179

Shapley, A. E., Steidel, C. C., Adelberger, K. L., et al. 2001, ApJ, 562, 95

Shapley, A. E., Steidel, C. C., Pettini, M., \& Adelberger, K. L. 2003, ApJ, 588,65

Shibuya, T., Kashikawa, N., Ota, K., et al. 2012, ApJ, 752, 114

Shimasaku, K., Kashikawa, N., Doi, M., et al. 2006, PASJ, 58, 313

Stark, D. P., Ellis, R. S., Chiu, K., Ouchi, M., \& Bunker, A. 2010, MNRAS, 408, 1628

Stark, D. P., Ellis, R. S., \& Ouchi, M. 2011, ApJL, 728, L2

Steidel, C. C., Giavalisco, M., Pettini, M., Dickinson, M., \& Adelberger, K. L. 1996, ApJL, 462, L17

Swinbank, J., Baker, J., Barr, J., Hook, I., \& Bland-Hawthorn, J. 2012, MNRAS, 422, 2980

Takahashi, M. I., Shioya, Y., Taniguchi, Y., et al. 2007, ApJS, 172, 456

Taniguchi, Y., Ajiki, M., Nagao, T., et al. 2005, PASJ, 57, 165

Tapken, C., Appenzeller, I., Noll, S., et al. 2007, A\&A, 467, 63

Teplitz, H. I., Collins, N. R., Gardner, J. P., Hill, R. S., \& Rhodes, J. 2003, ApJ, 589,704

Tilvi, V., Rhoads, J. E., Hibon, P., et al. 2010, ApJ, 721, 1853

Tran, K.-V. H., van Dokkum, P., Illingworth, G. D., et al. 2005, ApJ, 619, 134

Trenti, M., \& Stiavelli, M. 2008, ApJ, 676, 767

Vanzella, E., Pentericci, L., Fontana, A., et al. 2011, ApJL, 730, L35

Verdugo, T., de Diego, J. A., \& Limousin, M. 2007, ApJ, 664, 702

Verhamme, A., Schaerer, D., Atek, H., \& Tapken, C. 2008, A\&A, 491, 89

Verhamme, A., Schaerer, D., \& Maselli, A. 2006, A\&A, 460, 397 